Historic, Archive Document

Do not assume content reflects current scientific knowledge, policies, or practices. 
<smiles>C=CC</smiles> 


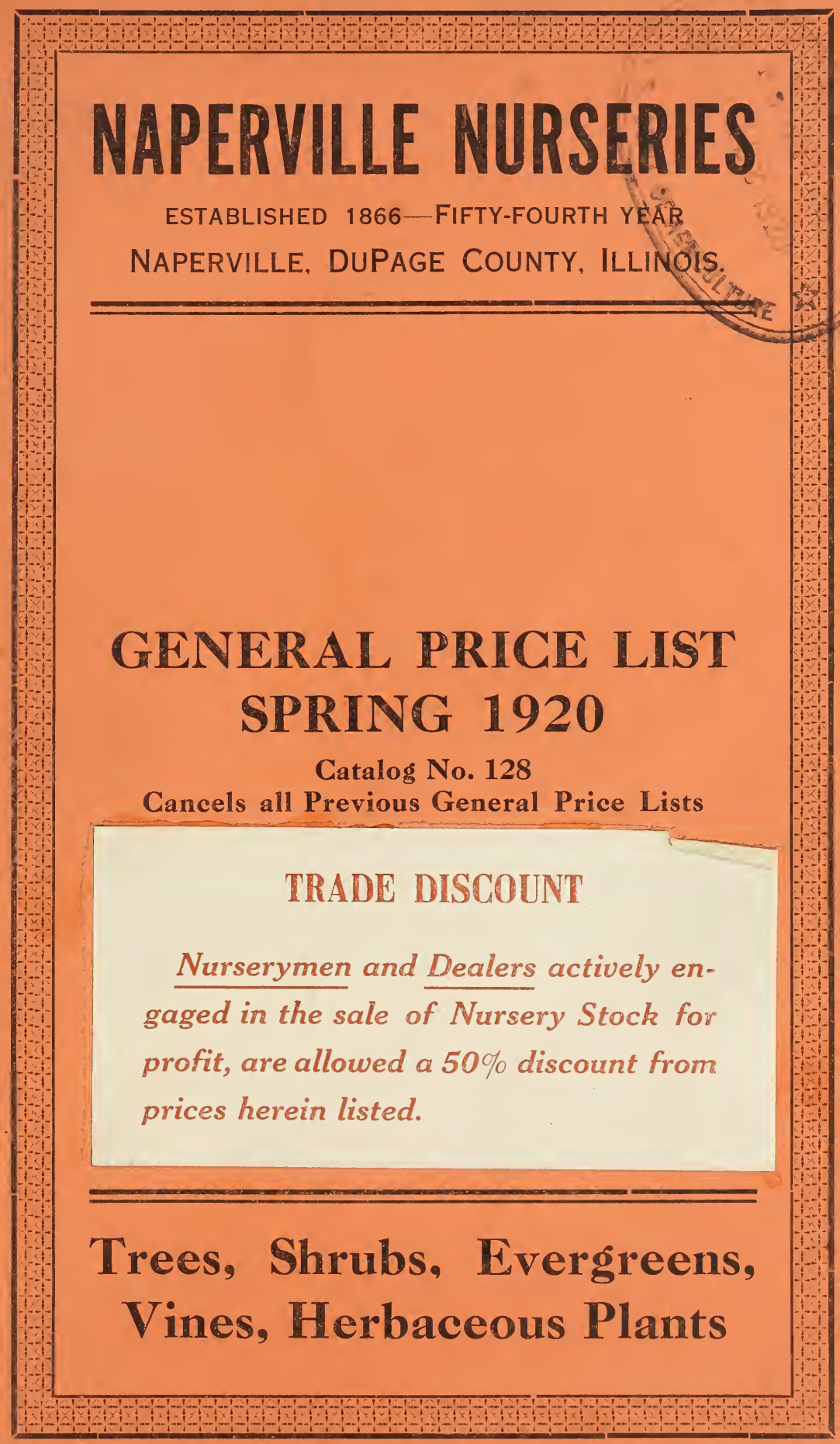





\title{
General Price List
}

\section{Spring 1920}

OF THE

\section{NAPERVILLE NURSERIES}

\author{
NAPERVILLE, ILLINOIS
}

PRICES HEREIN CANCEL ALL- PREVIOUS LISTS

\section{Advice to Correspondents, Directions to Customers and Conditions of Sale.}

\section{TERIMS AND CONDITIONS OF SALE.}

All prices named herein are,

a. Limited to Spring, 1920, and supercede those of all previous General Price Lists.

b. For goods f. o. b. Nurseries or cars Naperville, Illinois, exclusive of any packing, express, freight or transportation charges; packing charges are extra in addition to cost of plants.

c. Subject to cancellation or change without further notice upon prior sale or depletion of our stock of goods.

QUANTITY AND RATES: Single plants as well as lots amounting to less than 5 of a kind will be charged for at a higher rate not exceeding, however, $1 \frac{1}{2}$ times the rate for lots of 10 . Lots of 5 and 50 will be furnished at 10 and 100 rates respectively. Order in Tens and multiples of Ten of a variety as far as possible.

SELECTED STOCK, selected by customers at the Nurseries, will be charged for according to the value of the plants chosen.

$O R D E R S$. Write plainly and give explicit directions as to address and mode of shipment, whether by freight, express, etc.

Orders are accepted and all shipments made, subject to delays or inability to ship or deliver by reason or on account of strikes, accidents, droughts, fire, floods, frost, hail, sleet, war conditions and orders, railroad or express embargoes, priority or other transportation orders, prior sale of stock, car shortage, or any other cause or causes for delay in shipment or delivery of goods which are beyond our control.

Due to general conditions of weather, possible labor shortage and other contingencies, during the digging and shipping season, we do not guarantee to fill all items of an order or orders placed with us in one or first shipment

In making all shipments we carefully consider conditions in general and act accordingly.

GUARANTEE AND CLAIMS. . In growing and furnishing all Nursery stock, we exercise the utmost diligence and care to have it true to name, and will, on proper proof, to our satisfaction, replace any that will prove otherwise or refund the amount paid. Any and all sales made by us no not include any warranty expressed or implied, as to quality, description, productiveness, growth, or any other matter or qualifications pertaining to the Nursery stock sold.

It is mutually agreed and understood between the Purchaser and Ourselves, that in any case we shall not be liable for a sum greater than the amount originally paid us for said trees or plants, etc., that prove untrue. 
All shipments at purchaser's risk.

In absence of specific shipping instructions, we are to route all shipments according to our best judgment without ascsuming responsibility.

If destination is a Pre-Paid Station, freight charges will be prepaid by us and charged for on invoice.

Claims for errors or shortage must be filed with us within Ten (10) days after receipt of goods. No claims for errors or shortage in excess of purchase price will be considered.

Payments of accounts to be as follows:-

a. 30 days net cash, except Fall and Spring accounts with the trade, which are due on December 1st and June 1st respectively: $2 \%$ discount if paid within 10 days from date of shipment or invoice. For cash in advance a $3 \%$ cash discount is allowed.

b. All accounts after dates due, are subject to sight draft and interest charges at rate of $7 \%$ per annum, without further notice.

c. Payments for partial shipments shall be due on basis of dates of invoices covering them.

d. Cash or satisfactory references with order, if purchaser has no credit established with us.

Certificate of inspection will be futnished with each shipment.

Address all communications, orders and remittances to

NAPERVILLE NURSERIES, Naperville, DuPage County, Illinois.

Department of Agriculture. Division of Plant Industry. OFFICE OF CHIEF INSPECTOR.

No. 9.

Urbana, I11., Aug. 11, 1919.

This Is To Certify that the growing nursery stock and premises of Naperville Nurseries, situated at Naperville, Illinois, have been inspected by a duly appointed inspector of this office, according to the provisions of the Plant Inspection Act of 1917, and that said nursery and premises are apparently free from the San Jose Scale and other dangerous insects and dangerous plant diseases.

This certificate applies only to the condition of this nursery for the year ending August 11, 1920.

P. A. Glenn, Chief Inspector. Charles Adkins, Director.

\section{ORNAMENTAL SHADE TREES}

Each Per 10 Per 100

$A C E R$ dasycarpum, Native Silver Leaved or Soft Maple

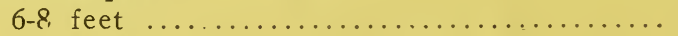

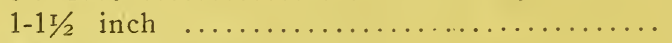

1.00

$9.00-80.00$

$2.25 \quad 17.50 \quad 150.00$

platanoides, Norway Maple

$11 / 2-2$ inch .............................. 4.00

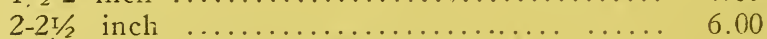

$35.00 \quad 300.00$

$55.00 \quad 450.00$

$21 / 2-3$ inch, Twice Transplanted... ........

7.50

600.00

$3-3 \mathrm{r} / 2$ inch, Twice Transplanted........... 10.00

$70.00 \quad 600.00$

$3 \mathrm{I} / 2.4$ inch, Twice Transplanted............ 18.00

90.00

$4-41 / 2$ inch, Twice Transplanted........... 20.00

150.00

$4 \mathrm{~L} / 2-5$ inch. Twice Transplanted.......... 30.00

170.00

6 inch, Price on application

240.00

Larger sizes, prices on application. 


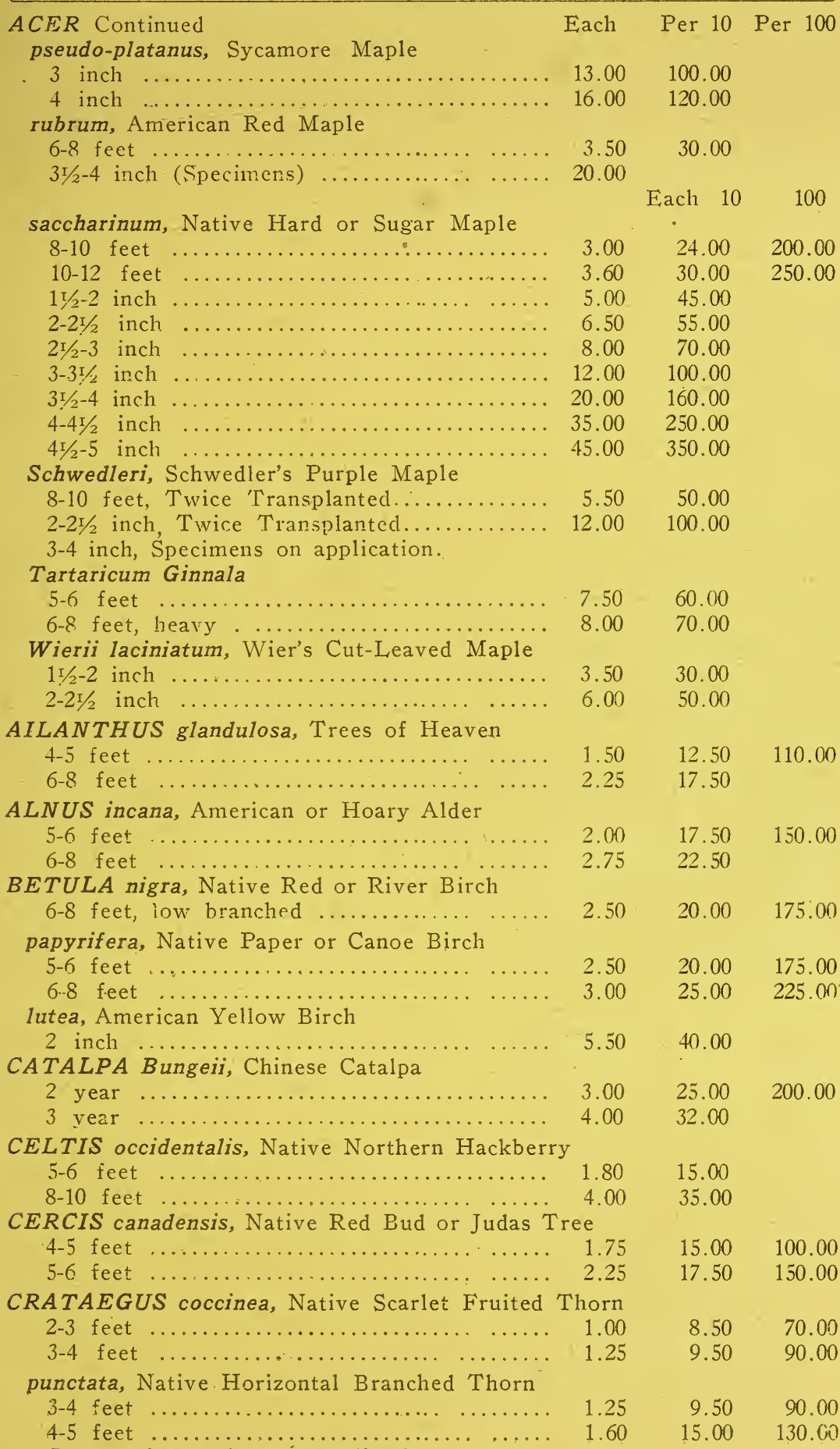

Larger sizes, prices on application. 
FAGUS Americana, American Beech

5-6 feet, low branched, B \& B (Specimens). 10.00

purpurea, Purple-leaved Beech

4-5 feet, low bránched, B \& P (Specimens) $9.00 \quad 80.00$

sylvatica, European Beech

5 feet, low branched, B \& B (Specimens)... $11.00 \quad 90.00$

FRAXINUS, American White Ash

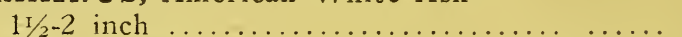

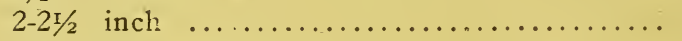

$2 \mathrm{r} / 2-3$ inch $\ldots \ldots \ldots \ldots \ldots \ldots \ldots \ldots \ldots \ldots \ldots \ldots \ldots \ldots \ldots \ldots \ldots \ldots$

2.75

25.00

$5.00 \quad 45.00$

$6.50 \div \quad 55.00$

Excelsior, European Ash

$2-2 \mathrm{r} / 2$ inch $\ldots \ldots \ldots \ldots \ldots \ldots \ldots \ldots \ldots \ldots \ldots \ldots \ldots \ldots$

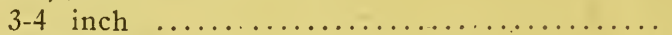

sambucifolia, American Black Ash

$1 \frac{1}{4}-11 / 2$ inch

$5.00 \quad 45.00$

$7.00 \quad 60.00$

2.50

22.00

180.00

viridis, American Green Ash

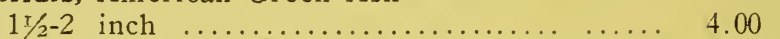

35.00

300.00

$2-2 \mathrm{r} / 2$ inch

5.00

45.00

55.00

$2 \mathrm{~T} / 2-3$ inch

6.50

8.00

65.00

GLEDITSCHIA triacanthos, Native Honey Locust

6-8 feet

$8-10$ feet $\ldots \ldots \ldots \ldots \ldots \ldots \ldots \ldots \ldots \ldots \ldots \ldots$

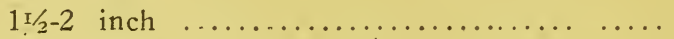

$2-21 / 2$ inch $\ldots \ldots \ldots \ldots \ldots \ldots \ldots \ldots \ldots \ldots \ldots \ldots$

$2 \mathrm{r} / 2-3$ inch

3.00

15.00

22.50

120.00

4.50

30.00

5.00

3-4 inch

7.00

10.00

var, Thornless

8-10 feet ............................. 3.00

45.00

60.00

25.00

GYMNOCLADUS canadensis, Kentucky Coffee Tree

8-10 feet ........................ 5.00

JUGLANS cinerea, Native Butternut

$5-7$ feet ....................... 1.30

nigra, Native Black Walnut

$4-5$ feet ............................ 1.20

6-8 feet ............................ 1.80

LARIX Americana, American Larch or Tamarack 5-6 feet, F. \& B .......................

8-10 feet, $B$ \& $B \ldots \ldots \ldots \ldots \ldots \ldots \ldots$

Europea, European Larch

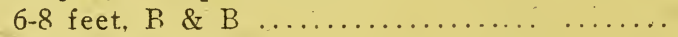

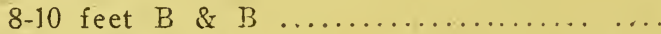

3.50

5.00

3.50

5.00

10.00

90.00

10.00

90.00

15.00

140.00

30.00

275.00

$45.00 \quad 450.00$

30.00

275.00

LIRIODENDRON tulipifera, American Tulip

Tree or Whitewood

$6-8$ feet $\ldots \ldots \ldots \ldots \ldots \ldots \ldots \ldots \ldots \ldots \ldots \ldots \ldots \ldots \ldots$

$1-11 / 2$ inch

20.00

30.00

160.00 250.00

MAGNOLIA acuminata, American Cucumber Tree

$5-7$ feet ........................... 2.50

20.00

$M A L U S$ (Pyrus)

floribunda, Japanese Pink Flowering Crab

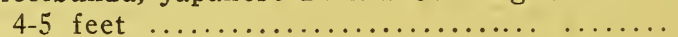

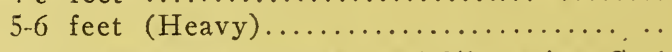

floribunda atrosanguinea, Red Flowering Crab

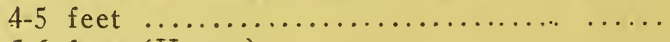

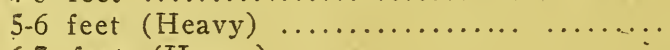

6-7 feet (Heavy)

100.00 
MALUS Continued

Ioensis, Native Wild Crab
Each

1.00

1.25

$3-4$ feet

Ioensis, var. Bechteli, Bechtel's Double

Pink Flowering Crab

2-3 feet

3-4 feet

Parkmanni, Parkman's Crab

5-6 feet (Heavy) ...................... 3.00

Scheideckeri, Double Flesh Colored Flowering Crab

$5-6$ feet (Heavy) ..................... 2.75

6-7 feet (Heavy) ................... 3.00

spectabilis, Chinese Pink Flowering Crab

$4-5$ feet ......................... 2.50

5-6 feet (Heavy) ..................... 3.50

PLATANUS occidentalis, American Sycamore

$1 \mathrm{i} / 2-2$ inch

\section{POPULUS}

alba Bolleana, Bolle's Silver Poplar

8-10 feet ........................... 3.00

balsamifera, American Balm of Gilead

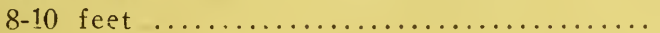

$10-12$ feet

1.00

1.50

monilifera, Cottonwood, Necklace Poplar

$6-\delta$ fect

8-10 feet

monilifera, var. Carolina, Carolina Poplar

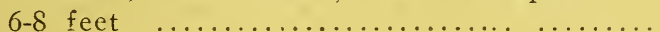

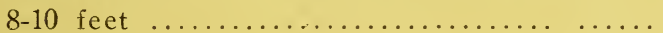

$1-1 \mathrm{x} / 2$ inch

$2-2 \mathrm{I} / 2$ inchi

nigra Italica, Lombardy Poplar

$1 \mathrm{I} / 2-2$ inch, I, ow Pranched ............... 2.50

$2-2 \mathrm{I} / 2$ inch, Low Brancled ............. 4.50

3 inch ........................... 7.50

2.00

2.50

.60

.70

1.50

3.00

3.00

$2-2 \frac{1}{2}$ inch

tremuloides, American Aspen

8-10 feet

3.50
8-10 feet

Per 10 Per 100

$8.00 \quad 70.00$

$10.00 \quad 90.00$

$12.50 \quad 100.00$

$15.00 \quad 130.00$

$25.00 \quad 225.00$

$22.50 \quad 200.00$

$25.00 \quad 225.00$

20.00

30.00

30.00

250.00

$42.50 \quad 380.00$

$25.00 \quad 200.00$

$8.00 \quad 70.00$

12.00

15.00

20.00

5.00

40.00

$6.00 \quad 50.00$

$12.50 \quad 100.00$

$27.50 \quad 250.00$

$20.00 \quad 175.00$

$35.00 \quad 300.00$

60.00

25.00

30.00

250.00

PRUNUS Pennsylvanica, Native Wild Red or Pin Cherry $6-8$ feet $\ldots \ldots \ldots \ldots \ldots \ldots \ldots \ldots \ldots \ldots \ldots \ldots . . . \ldots \ldots$

serotina, American Wild Black Cherry

3-4 feet .............................. . . 70

60.00

4-5 feet

.80

5.00

40.00

6.00

50.00

Virginica, Native Choke Cherry

3-4 feet ........................... .80

6.00

50.00

4-5 feet

.90

7.00

60.00

PTELIA trifoliata, Native Hop Tree

6-8 feet

1.50

12.00

100.00

PYRUS (Flowering Crab), (see Malus)

QUERCUS alba, Native White Oak

$5-6$, feet

$1-1 \frac{1}{2}$ inch

5.00

45.00

macrocarpa, Native Burr Oak

3-4 feet

12.50

100.00 
QUERCUS Continued

palustris, Native Pin Oak

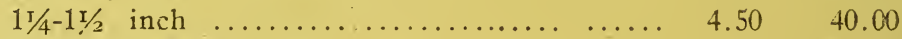

rubra, Native Red Oak

Each

4.50

inctoria, Native Black Oak

3-5 feet

OBINIA pseudo-acacia, American Black Locust

4-5 feet

5-7 feet

SALIX alba, American White Willow

4-6 feet

Babylonica Dolorosa, Wisconsin Weeping Willow

4-5 feet ............................ 80

discolor, Pussy Willow

3-4 feet, 1 year ....................... .40

laurifolia, European Laurel-leaved Willow

$4-5$ feet ......................... $\quad .80$

viminalis, Basket Willow

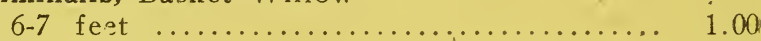

8-10 feet ............................... 1.20

vitellina, var. Britzensis, Bronze Barked Willow

3-4 feet ............................ .80

SYRINGA Chinensis, Chinese Tree Lilac

3 feet ........................... 1.50

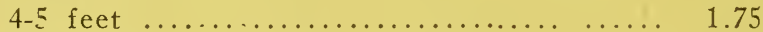

Japonica, Japan Tree Lilac, $3-4$ feet..........

TAXODIUM distichum, American Bald Cypress

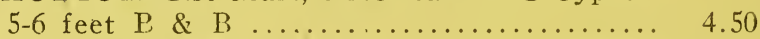

TILIA Americana, American Linden or Basswood

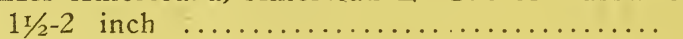

$2-21 / 2$ inch $\ldots \ldots \ldots \ldots \ldots \ldots \ldots \ldots \ldots \ldots \ldots \ldots \ldots$

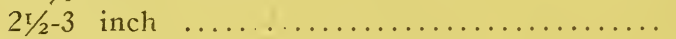

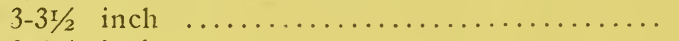

$31 / 2-4$ inch $\ldots \ldots \ldots \ldots \ldots \ldots \ldots \ldots \ldots \ldots \ldots \ldots \ldots$

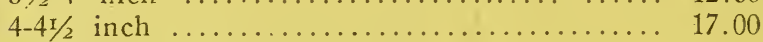

platyphyllos, European Linden

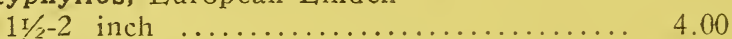

ULMUS Americana, American Elm

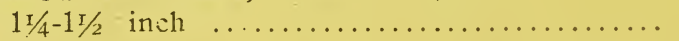

$11 / 2-2$ inch

2.30

3.50

$2-2 \mathrm{I} / 2$ inch

6.00

$2 \mathrm{t} / 2-3$ inch

$3-3 \mathrm{~T} / 2$ inch

8.00

10.00

13.00

Select or Larger sizes, prices on application. campestris, English Elm

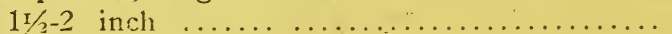

$2 \mathrm{I} / 2-3$ inch ............................. 7.50

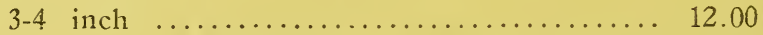

WEEPING OR PENDULOUS TREES

$A C E R$ Wierii laciniatum, Wier's Cutleaved Maple

$11 / 2-2$ inch .......................... 3.50

30.00

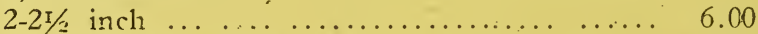

50.00

BETULA alba pendula, Weeping Birch

6-8 feet heavy, B \& B ................ $6.00 \quad 50.00$

alba var. laciniata (Cut-leaved Birch

6-7 feet
50.00

60.00

$5.00 \quad 40.00$

$6.00 \quad 50.00$

$3.50 \quad 20.00$

$6.00 \quad 50.00$

7.00

60.00

80.00

$7.00 \quad 60.00$

10.00

12.50

10.00

40.00

35.00

50.00

60.00

90.00

120.00

150.00

35.00

20.00

180.00

$30.00-280.00$

$50.00 \quad 500.00$

$70.00 \quad 600.00$

110.00

40.00

60.00

90.00 


\begin{tabular}{|c|c|c|c|}
\hline MORUS Tartarica pendula, Teas Weeping & Each & Per 10 & Per 100 \\
\hline $\begin{array}{cc}\text { Mulberry } & 2 \text { year head ................. } \\
\text { SALIX Babylonica dolorosa, Wisconsin Weeping }\end{array}$ & 4.00 & 35.00 & \\
\hline $\begin{array}{l}\text { Willow } 4-5 \text { feet } \ldots \ldots \ldots \ldots \ldots \ldots \\
\text { ULMUS montana pendula, Camperdown Elm }\end{array}$ & .80 & 6.00 & 50.60 \\
\hline 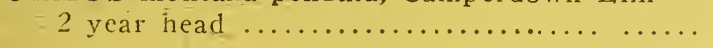 & 4.00 & 35.00 & \\
\hline
\end{tabular}

\section{FRUITS}

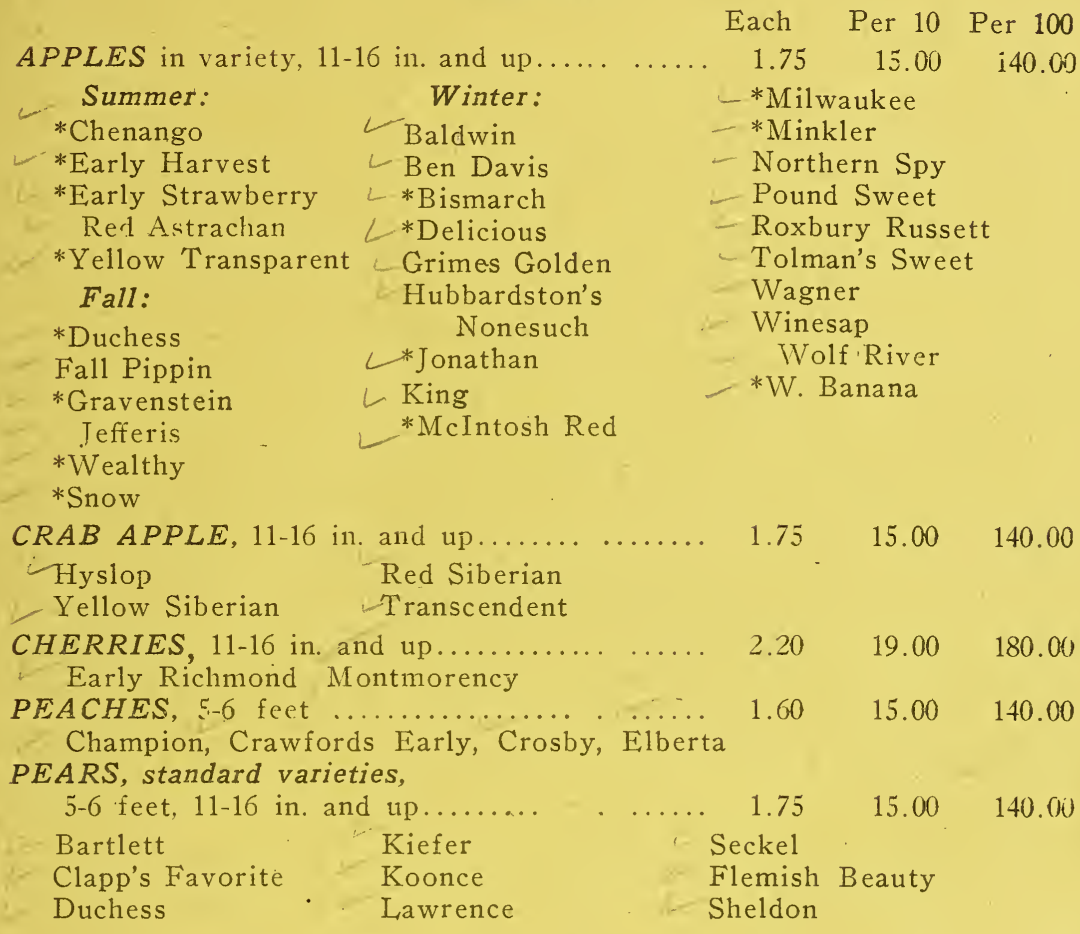

$\begin{array}{lllll}\text { Dwarf varieties, } 5-8 \text { feet and up ............ } & 1.50 & 13.00 & 110.00\end{array}$

$\checkmark$ Bartlett, Duchess, Flemish Beauty, Seckel

PLUMS, American varieties, 5-6 ft., 11-16 in.

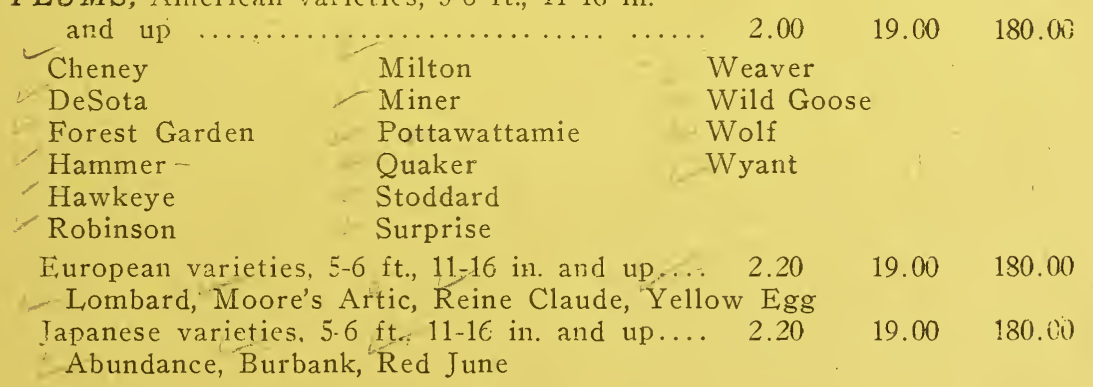




\section{SMALL FRUITS.}

BLACKBERRIES, Mercereau, Snyder'\$ Each Per 10 Per 100 2 year ................................ $15.75 \quad 15.00$ CURRANTS, Black, Red, White,
$7.50 \quad 60.00$

GOOSEBERRIES, Downing, Houghton, Red Jacket,

Transparent

2 year

7.50

60.00

GRAPES

2 year

.80

$7.00 \quad 60.00$

1 year .70

$6.00-50.10$

$\begin{array}{lll}\text { Deleware } & \text { L Niagara } & \text { Concord } \\ \text { Worden } & \text { - Brighton } & \text { Martha } \\ \text { Agawan } & \text { Moore's Early } & \\ \text { Diamond } & \text { Mocklington } & \end{array}$

\section{RASPBERRIES}

"Kansas, Gregg, Cumberland, 1 year......... .20

Red,"Columbia, Louden, St. Regis, 1 year... .25

Red, 1 year, LColumbian................. .20

$\begin{array}{ll}1.50 & 10.00 \\ 1.60 & 12.00 \\ 1.50 & 10.00\end{array}$

STRAWBERRIES in variety. Bederwood,

4 Nick Olimer. Haverland, Senatodr Dunlap-\$20.00 per 1000 . 3.50

$\checkmark$ Superb and Progressive, $\$ 40.00$ per 1000

ASPARAGUS, 2 year- $\$ 25.00$ per 1000.

Bonvallet's Giant, Conover's Colossal,

Early Argenteil, Palmetto

\section{EVERGREENS}

Never fail to properly mulch all Evergreen Plantings whenever planted. Hot and cold water are dangerous when applied to ground directly around Roots.

ABIES Balsamea, American Balsam Fir

Each Per 10

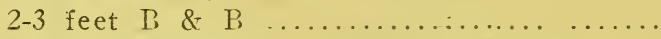

$4.00 \quad 35.00$

Price on larger sizes made on application.

Tsuga Canadensis, American or Northern Hemlock

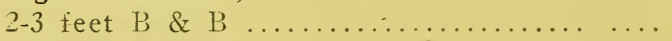

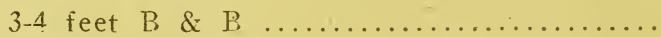

$5.00^{-} \quad 45.00$

$7.00 \quad 60.00$
$10.00-90.00$

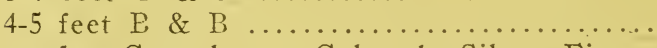

$10.00 \quad 90.00$

concolor, Concolor or Colorado Silver Fir

$2-2 \mathrm{r} / 2$ feet $\mathrm{B} \& \mathrm{~B} \ldots \ldots \ldots \ldots \ldots \ldots \ldots . \ldots . \ldots . \ldots . \ldots$

$6.00 \quad 55.00$

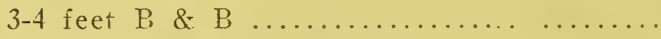

$8.50 \quad 75.00$

Sizes 8-16 feet or Specimens-Prices on application.

Douglasii, Douglas Spruce

$2-3$ feet $E$ \& $B \ldots \ldots \ldots \ldots \ldots \ldots \ldots \ldots \ldots \ldots \ldots \ldots$

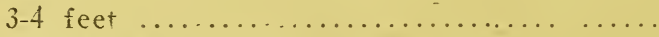

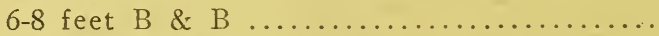

JUNIPERUS, Canadensis var. depressa. Native or

$4.50 \cdot 37.50$

$6.00 \quad 50.00$

$16.00 \quad 150.00$

Canadian Juniper

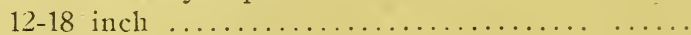

Each Per 10

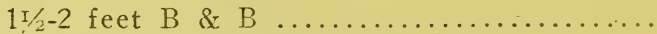

$3.50 \quad 30.00$

$5.50 \quad 47.50$

Communis Suecciafi Swedish Juniper

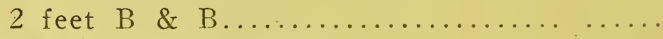

$3.50-27.50$

hibernica, Irish Juniper

2-3 feet B \& $\mathrm{B}$.

$3.50 \quad 25.00$

sabina, Savin Juniper

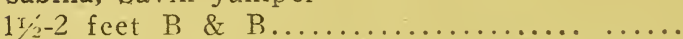

$6.00 \quad 50.00$ 
JUNIPERUS Continued

var Tamariscifolia

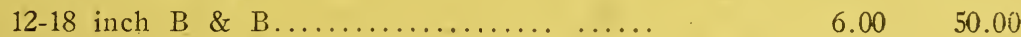

$2-2 \mathrm{r} / 2$ feet B \& B..................... $10.00 \quad 85.00$

Virginiana, Native Red Cedar

$3-4$ feet $\mathrm{P} \&$ \& B ....................... $\quad 6.00 \quad 50.00$

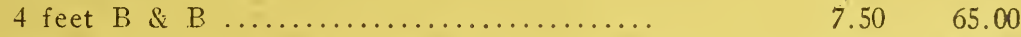

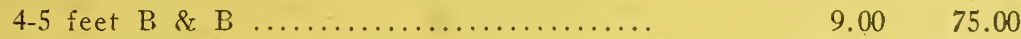

5 feet $B \&$ B ................................ $11.50 \quad 100.00$

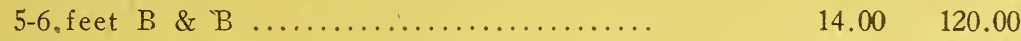

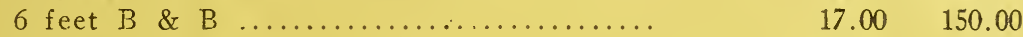

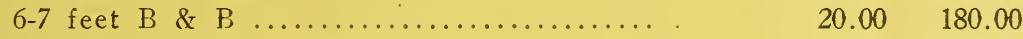

7-8 feet B \& B ..................... $30.00 \quad 250.00$

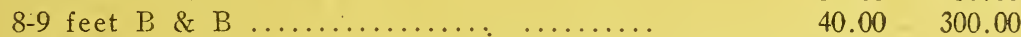

Prices on larger sizes and Specimens made upon application.

PICEA Canadensis, Black Hills Spruce

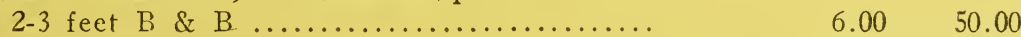

$3-4$ feet $B$ \& B ........................ $\quad 8.50 \quad 75.00$

Excelsa, Norway Spruce

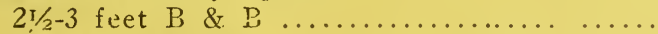

$3.50 \quad 27.50$

$3-4$ feet $B$ \& $-B \ldots \ldots \ldots \ldots \ldots \ldots \ldots \ldots \ldots \ldots \ldots \ldots$

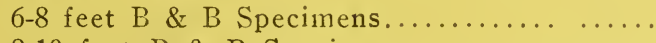

8-10 feet B \& B Specimens................

$4.50 \quad 35.00$

$15.00 \quad 125.00$

Larger Sizes or Specimens-Prices on application.

$20.00 \quad 175.00$

pungens, Colorado Blue Spruce

$2-21 / 2$ feet $B$ \& $B \ldots \ldots \ldots \ldots \ldots \ldots \ldots \ldots \ldots$

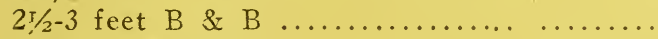

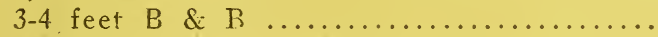

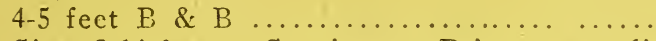

$8.00 \quad 70.06$

$9.00 \quad 80.00$

$12.00 \quad 100.00$

Sizes 8-14 feet or Specimens-Prices on application.

$15.00 \quad 140.00$

Green Type.

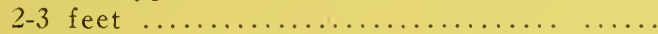

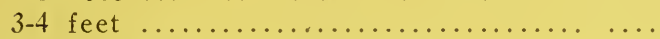

PINUS Austriaca, Austrian Pine

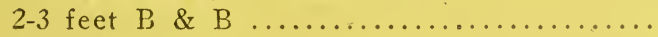

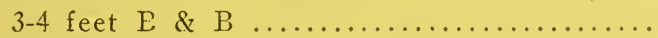

$8.50 \quad 75.00$

$+.50 \quad 35.00$

$7.50 \quad 70.00$

Banksiana, American Jack Pine

3-4 feet B \& $B$........................ $4.00 \quad 45.00$

Mugho Pine, Dwarf Mountain Pine

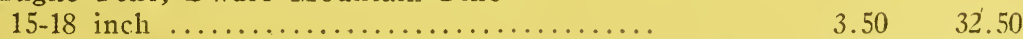

$18-24$ inch .............................. 4.50 .040

ponderosa, Bull Pine

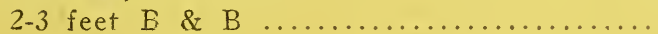

$4.00 \quad 30.00$

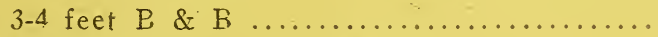

$5.00 \quad 40.00$

Resinosa, Red or Norway Pine

$2-3$ feet B \& B ......................

$4.00 \quad 32.50$

strobus, Native White Pine

3.4 feet $B \& B$. $B \ldots \ldots \ldots \ldots \ldots \ldots \ldots \ldots \ldots$.

$4.75 \quad 40.00$

$5.50 \quad 44.50$

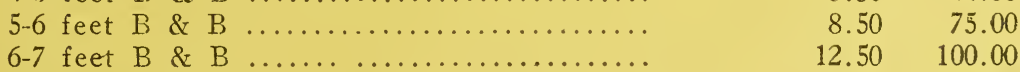

$7-8$ feet $B$ \& $B$..................... $\quad 17.50 \quad 150.00$

Larger sizes, Prices on application.

sylvestris, Scotch Pine

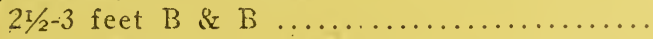

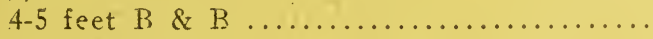

$5-6$ feet $B$ \& $B \quad \ldots \ldots \ldots \ldots \ldots \ldots \ldots \ldots$. 
THUYA occidentalis, American Arbor Vitae

$\begin{array}{rr}\text { Each } & \text { Per } 10 \\ 3.00 & 25.00 \\ 4.50 & 35.00 \\ 5.50 & 45.00 \\ 4.00 & 38.00 \\ 3.50 & 30.00 \\ & \\ 5.50 & 45.00 \\ 7.50 & 65.00\end{array}$

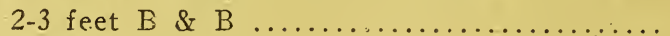

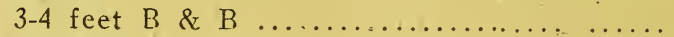

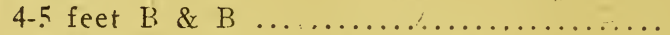

globosa, Dwarf Globe Arbor Vitae

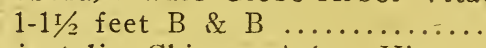

orientalis, Chinese Arbor Vitae

$2-3$ feet ............................

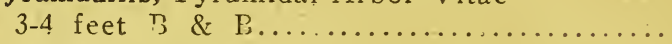

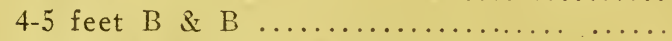

Larger sizes, Prices on Application.

\section{SHRUBS}

ACANTHOPANEX pentaphyllum, Aralia.

(See Aralia pentaphylla)

$A L M O N D$ (See Prunus sinensis.)

ALTHEA, Rose of Sharon. In variety

Boule de Feu

Elegantissima

Joan d'Arc

Lady Stanley

Peonoflora

3-4 feet

60.00

AMELANCHIER alnifolia, Dwarf June Berry

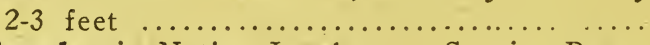

$6.00 \quad 50.00$

Canadensis, Native Juneberry. Service Berry

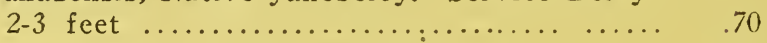

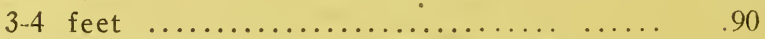

$6.00 \quad 50.00$

$7.00 \quad 60.00$

ARALIA pentaphylla, Five Leaved Aralia

2-3 feet

40.00

Mandschurica, Mandschurian Aralia

4-5 feet

1.25

spinosa, American Hercules Club

3-4 feet

ARONIA floribunda, American Red Choke Berry

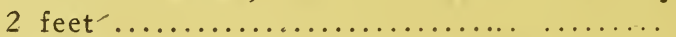

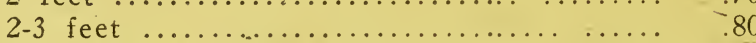

arbutifolia, var. nigra, American Black Choke Berry

18 inch ............................. $\quad .70$

BERBERIS Thunbergii, Thunberg's Japanese Barberry

$15-18$ inch Bushy ..................... $\quad .40$

$18-24$ inch ............................ .50

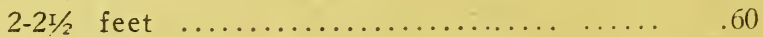

$B U D D L E Y A$ variabilis, Butterfly Bush

2 year ........................... 70

CALYCANTHUS floridus Carolina, American All-Spice,

Sweet scented shrub

3-4 feet ........................... .70

CARAGANA arborescens, Siberian Pea Tree Each

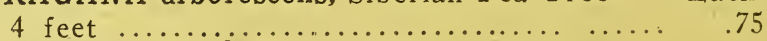

CEANOTHUS americanus, New Jersey Tea

$12-18$ inch .......................... .80

CEPHALANTHUS occidentalis, Native Button Bush

2-3 feet

.70

$5.00 \quad 45.00$

Per 10 Per 100

$6.00 \quad 50.00$

$6.00 \quad 50.00$

$5.00 \quad 40.00$ 
Each Per 10 Per 100

CHIONANTHUS virginica, American White Fringe Tree

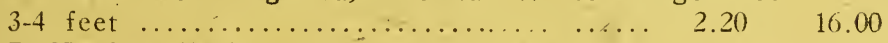

CLETHRA alnifolia, American Sweet Pepper Bush

$$
2-3 \text { feet ............................. } 70
$$

CORCHOROUS Japonica, Single Flowered Corchorous

$12-18$ inch .......................... .70

Japonica, variegata, Variegated leaved Corchorous $12-18$ inch ............................ .75

CORNUS alba, Native Red Dogwood

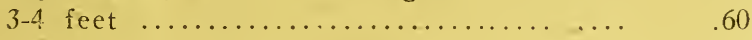

$4-5$ feet $\ldots \ldots \ldots \ldots \ldots \ldots \ldots \ldots \ldots \ldots \ldots . . .60$

$$
5.00
$$

$6.00 \quad 50.00$

$6.00 \quad 50.00$

alternifolia, Native Blue or alternate-leaved Dogwood $2-3$ feet .......................... $\quad .75$

florida, Native White Flowered Dogwood

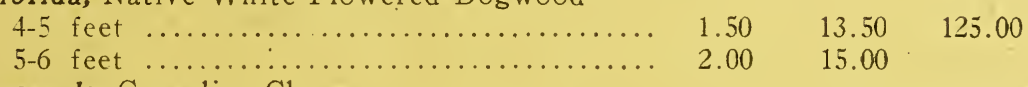

mascula, Cornelian Cherry

$2-3$ feet $\ldots \ldots \ldots \ldots \ldots \ldots \ldots \ldots \ldots \ldots \ldots \ldots \ldots$
paniculata, Native Panicled Cornel or Gray Dogwood

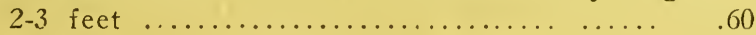

3-4 feet ........................... $\quad .80$

$6.00 \quad 50.00$

$5.00 \quad 40.00$

$7.00 \quad .60 .00$

sanguinea, English Osier.Dogwood

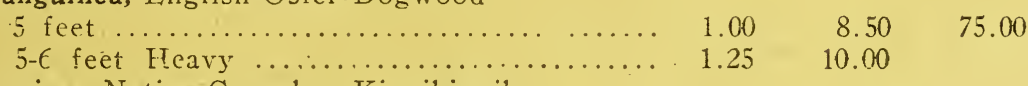

sericea, Native Cornel or Kinnikinnik

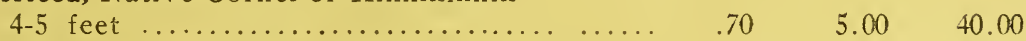

Siberica, Red Siberian Dogwood

3-4 feet .............................. $\quad .60$

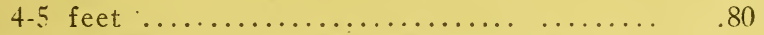

stolonifera, Native Red Osier Dogwood

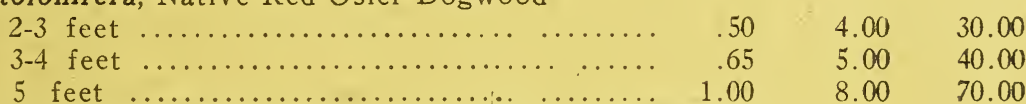

stolonifera var. flaviramea, Yellow Branched Dogwood $2-3$ feet ............................ 70

CORYLUS

\begin{tabular}{|c|c|c|}
\hline $2-3$ feet $\ldots \ldots \ldots \ldots \ldots$. & 1.00 & 8.50 \\
\hline $3-4$ feet $\ldots \ldots \ldots \ldots \ldots \ldots \ldots \ldots \ldots \ldots \ldots$ & 1.25 & $9: 50$ \\
\hline 4-5 feet & 1.60 & 15.00 \\
\hline -6 feet (Heavy) & 3.50 & 30.00 \\
\hline B \& B... & 10.00 & \\
\hline tive Horizontal Branched & 1.20 & 10.00 \\
\hline 5 feet $\ldots \ldots \ldots \ldots \ldots \ldots \ldots \ldots \ldots \ldots$ & 1.60 & 15.00 \\
\hline et (Heavy) & 3.50 & 30.00 \\
\hline
\end{tabular}

avellana, European Hazel

$5-8$ feet .......................... 2.00

CRATAEGUS coccinea, Native Scarlet Fruited Thorn

DESMONDIUM, (See Lespedeza)

DEUTZIA crenata, Pride of Rochester, large Each flowered Deutzia (Double White)

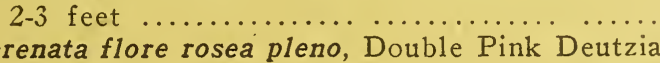

$2-3$ feet

Per 10 Per 100

gracilis, Slender Deutzia (Dwarf White)

$1 \mathrm{~T} / 2-2$. feet

$.60 \quad 4.00 \quad 35.00$

$\begin{array}{lll}.75 & 6.00 \quad 50.00\end{array}$ 
DEUTZIA Continued

Each

lemoinei, Lemoine's Deutzia (Pure White)

2-3 feet

DIERVILLA sessilifolia, Native Mountain

Honeysuckle

3-4 feet

trifida, Native Bush Honeysuckle (Dwarf)

12-15 inches

ELAEAGNUS, angustifolia, Russian Olive

3-4 feet

4-5 feet

5-6 feet

EUONYMUS, alatus, Winged Burning Bush

or Corked Barked Euonymus

3-4 feet

Americanus, Native Strawberry Bush

3-4 feet

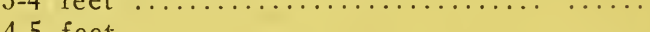

tropurpureus, Native Buring Bush or Wahoo

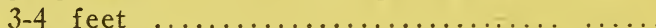

FORSYTHIA Fortuneii, Fortune's Golden Bell

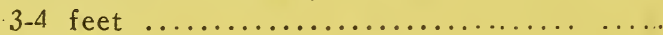

4-5 feet

intermedia, Hybrid Golden Bell

2-3 feet

3-4 feet

4 feet

suspensa, Drooping Forsythia

2-3 feet

3-4 feet

viridissima

4-5 feet

HAMAMELIS, Virginiana, Native Witch Hazel

3-4 feet

HYDRANGEA arborescens, Native Wild Hydrangea

2-3 feet ............................ . .70

arborescens sterilis, Hills of Snow

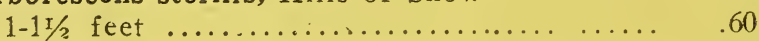

2-3 feet

paniculata grandiflora, Large Flowered

Hydrangea

18-24 inches

paniculata grandiflora, (Tree form)

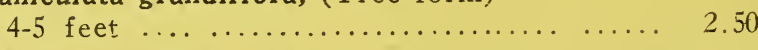

ILEX verticillata, American Black Alder or Winterberry

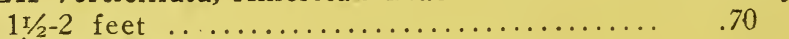

LESPEDEZA bicolor, Japanese Bush Clover

3-4 feet .............................. 80

LIGUSTRUM Amurense, Amoor River Privet (North Var.)

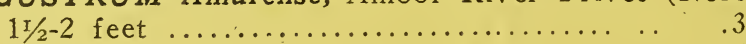

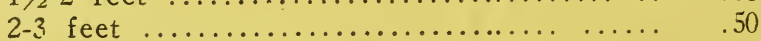

Ibota, Ibota Privet, Chinese Privet

$2-3$ feet ..............................5

Ibota Regelianum, Regel's Privet (true type)

$1 \mathrm{I} / 2-2$ feet

$2-3$ feet
Per 10 Per 100

6.50

60.00

80

7.00

60.00

60

5.00

40.00

5.50

45.00

$8.00 \quad 70.00$

12.00

100.00

1.25

10.00

90.00

12.50

10.00

90.00

60

5.00

35.00

$7.00 \quad 50.00$

3.50

30.00

$5.00 \quad 40.00$

6.00

50.00

60

5.00

40.00

6.00

50.00

$5.00 \quad 40.00$

9.00

80.00

$6.00 \quad 50.00$

$5.00 \quad 40.00$

$6.50 \quad 55.00$

4.50

40.00

20.00

$6.00 \quad 50.00$

$6.00-50.00$

$35 \quad 3.00$

20.00

$4.00 \quad 30.00$

50

$3.50 \quad 30.60$ 
LIGUSTRUM Continued vulgare, European Privet or Prim

2-3 feet

3-4 feet

LINDERA benzoin, American Spice Bush

$2-3$ feet

3-4 feet

LONICERA

bella albida, White Upright Honeysuckle, very rapid, upright grower, possessing an excellent foliage, makes a fine hedge plant

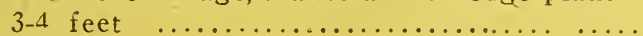

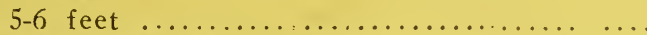

bella chrysantha, a very-fine plant of moderate growth, good habits and an excellent foliage. Little used because because its qualities are

little known

3-4 feet

grandiflora, Pink Honeysuckle

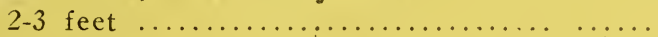

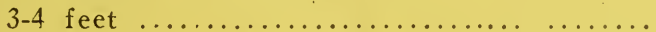

4-5 feet

5.00

8.00

40.00

70.00

Morrowi, Japanese Bush Honeysuckle

3-4 feet

4-5 feet

6-8 feet

tartarica rosea, Tartarian Pink Honeysuckle

3-4 feet ........................... 70

LYCIUM Chinense, Matrimony Vine or Box Thorn

2 year

MORUS Siberica, Russian Mulberry (Bush Form)

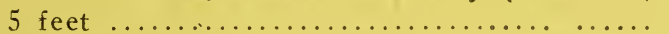

Per 10 Per 100

40.00

45.00

55.00
$4.00 \quad 30.00$

PHILADELPHUS coronarius, Common Mock Orange or Sweet Scented Syringa

3-4 feet ............................... .70

$4-5$ feet $\ldots \ldots \ldots \ldots \ldots \ldots \ldots \ldots \ldots . \ldots \ldots . . .60$

6.50

50.00

Gordonii, Gordon's or Western Mock Orange

3-4 feet ........................... $\quad .60$

grandiflorus, Large-Flowered Native Mock Orange

3-4 feet ........................... .60

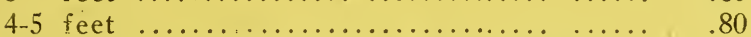

$4.50 \quad 36.00$

$4.50 \quad 36.00$

$6.50 \quad 50.00$

Lemoinei, Lemoine's Hybrid Mock Orange

3-4 feet

nivalis

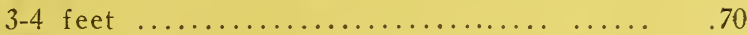

4 feet

Zeyherii, Zeyher's Mock Orange

$4-5$ feet $\ldots \ldots \ldots \ldots \ldots, \ldots \ldots \ldots \ldots \ldots \ldots \ldots \ldots \ldots, \quad .60$

$6.00 \quad 50.00$

POTENTILLA fruticosa, American Shrubby Cinquefoil

$11 / 2-2$ feet .......................... $\quad 60$

$4.50 \quad 40.00$

PRUNUS Americana, Native Wild Plum

3-4 feet

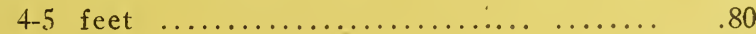

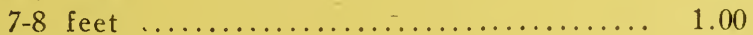

7.00

60.00

padus, Bird Cherry

4 feet 
- PRUNUS Continued

Each

pumila, Native Dwarf or Sand Cherry

2-3 feet

sinensis, fl. pl. var. alba, White Flowering Almond

$2-2 \mathrm{r} / 2$ feet $\ldots \ldots \ldots \ldots \ldots \ldots \ldots \ldots \ldots \ldots \ldots . . . .70$

sinensis, fl. pl. var. rosea, Pink Flowering Almond

$2-3$ feet $\ldots \ldots \ldots \ldots \ldots \ldots \ldots \ldots \ldots \ldots \ldots . . . . . . .60$

Per 10 Per 100

triloba, Double Flowering Plum

$2-3$ feet $\ldots \ldots \ldots \ldots \ldots$
$3-\ldots \ldots \ldots \ldots$

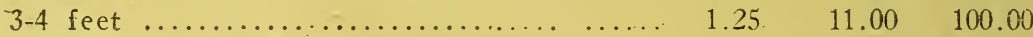

Virginiana, Native Choke Cherry

(Listed under Trees)

PYRUS arbutifolia. (See Aronia)

malus. (See Malus, under Trees)

RHAMNUS cathartica, European or Common

Thorned Buckthorn

3-4 feet ..............................60

frangula, American Thornless or Alder Buckthorn

4-5 feet ............................. 70

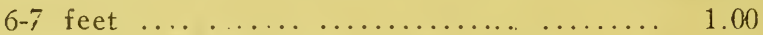

RHODOTYPOS kerrioides, White Kerria

2-3 feet ............................ .70

RHUS aromatica, Native Sweet-scented Sumach

2-3 feet

80

copallina, Native Dwarf or Shining Sumach

2-3 feet

36.00

$6.00 \quad 50.00$

$9.00 \quad 80.00$

cotinus, Smoke Bush or Purple Fringe

2-3 feet

.80

$6.00 \quad 55.00$

glabra, Native Smooth Sumach

4-5 feet

$6.50 \quad 55.00$

glabra var. laciniata, Cut-leaved Smooth Sumach

$1-11 / 2$ feet .......................... .80

typhina, Native Staghorn Sumach

3-4 feet ........................... 60

$4-5$ feet ........................... $\quad .70$

$6.50 \quad 55.06$

$65-5.00 \quad 45.00$

typhina, var. laciniata, Cut-leaved Staghorn

Sumach

$3-4$ feet $\ldots \ldots \ldots \ldots \ldots \ldots \ldots \ldots \ldots \ldots \ldots \ldots \ldots \ldots \ldots . . .70$

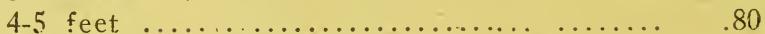

RIBES alpinum, Mountain Currant

$2-3$ feet $\ldots \ldots \ldots \ldots \ldots \ldots \ldots \ldots \ldots . \ldots . . . . . .60$

$4.50 \quad 35.06$

$5.50 \quad 40.00$

aureum, Missouri or Buffalo Currant

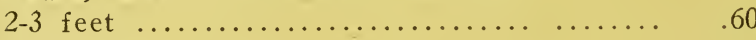

4-5 feet $\ldots \ldots \ldots \ldots \ldots \ldots \ldots \ldots \ldots \ldots \ldots \ldots \ldots . . .60$

$5.00 \quad .40 .00$

$6.00-50.00$

cynosbati, Native Gooseberry

3 feet

$6.50 \quad 56.00$

$5.00 \quad 45.00$

$7.00 \quad 60.00$

$7.00 \quad 60.00$

\section{ROSES}

BUSH

AMERICAN OR NATIVE

ROSA blanda, Meadow Rose

Each Per 10 Per 100

2-3 feet

$\begin{array}{lll}.60 & 5.00 & 40.00\end{array}$

Carolina, Swamp or River Rose

$2-2 \mathrm{r} / 2$ feet $\ldots \ldots \ldots \ldots \ldots \ldots \ldots$

$5.00 \quad 40.00$

humilis,
3 feet

5.00

40.00 
ROSA Continued

lucida, Upright Glossy Swamp Rose

Each Per 10 Per 100

$2-2$
itida, Dwarf Prickley, Glossy Swamp Rose

$.60-5.00 \quad 40.00$

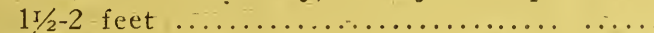

$\begin{array}{lll}.50 & 4.00 \quad 36.00\end{array}$

setigera, Illinois Prairie Rose (See Climbing

Roses) 2 year

3 yr.. No $1,4-5$ feet $. . . \ldots \ldots \ldots \ldots \ldots . . . . .$.

$6.50 \quad 60 .(0)$

\section{CLIMBING ROSES.}

Crimson Rambler, Double Crimson

Dorothy Perkins, Very Dark Pink, (One of the Best)

Evergreen Gem, Single

Excelsa, (Red Dorothy Perkins, Extra Fine)

Hiawatha, Single Bright Crimson

Lady Gay, Double Delicate Cherry Pink and Soft White

Prairie Queen, Double Bright Rosy Red

Setigera, (Illinois Prairie Rose)-Single, Rose Colored

Seven Sisters, Double Crimson, Changing to Lighter Shades

Tausendschon

White Dorothy Perkins, Double, Pure White

White Rambler, Small Double, Pure White

Wichuriania, Var. rubra, Memorial Rose, Single Red

2 year, No. 1 ........................ 1.00

$9.00 \quad 80.00$

HYBRID TEAS.

Arthur S. Goodwin, Rich Coppery Orange

Duchess of Wellington, Saphryon Yellow

Edward Mawley, Rich Crimson..

Florence Pemberton, Creamy White Suffused with pink

Gruss an Teplitz, Velvety Crimsson

General McArthur, Warm Rich Crimson Scarlet

James Hill, Salmon Pink

Geo. C. Waud, Glowing Vermillion With Orange Red Suffusion

Killarney, Brilliant Rich Rosy Carmine

Kaiserin Augusta Victoria, Soft Pearly White

Lyon, Salmon Pink, Shaded with Yellow in Center, Shrimp Pink at the Tips

Maryland, Salmon Pink

Mad. Caroline Testout, Bright Satin

White Killarney

Mme. Able Chatenay, Carmine Rose with Salmon Shading

Mrs. Geo. Shawyer, Brilliant Clear Rose

Sunburst, Rich Cadmium Yellow

Mad. Ravary, a Distinct Shade of Nankeen Yellow

Mrs. Wakefield Christie Miller, Bright Pink Flower with Crisp Gold.

2 year, No. i ....................... $2.00 \quad 15.00 \quad 120.00$

\section{JAPAN.}

ROSA multiflora, Japanes̀e Climbing Rose.

(See Climbing Roses)

rugosa alba, White Japanese Wrinkled Rose

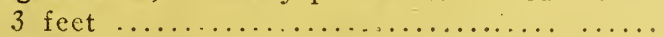

rugosa rubra, Red Japanese Wrinkled Rose

2-3 feet

60.00

ROSA rubiginosa, Sweet Briar

$\begin{array}{lll}.70 & 6.00 \quad 50.00\end{array}$

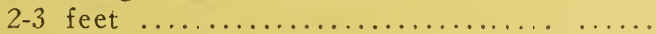

3-4 feet .............................. $\quad .80$ 
RUBUS Continued odoratus, Native Purple Flowering Raspberry

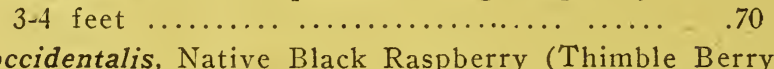

Each

Per 10 Per 100

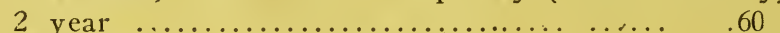

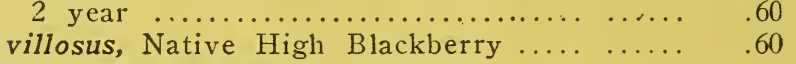

SAMBUCUS aurea, Golden Elder

4-5 feet ............................. 80

Canadensis, American Common Elder

.80

5.00

5.00

40.00

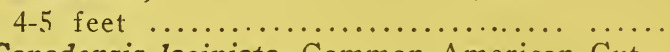

.70

$6.00 \quad 55.00$

Canadensis laciniata, Common American Cut-

leaved Elder

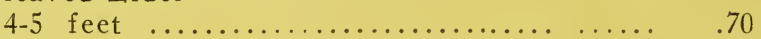

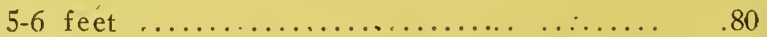

$5.50 \quad 40.01)$

pubens, American Red-berried Elder

3-4 feet .............................. . . . .

5-6 feet (Heavy) ...................... 1.20

$6.00 \quad 40.00$

$7.00 \quad 50.00$

SPIREA Anthony Waterer, Crimson Spirea

$12-18$ inches .......................... $\quad 60$

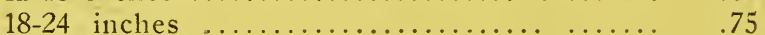

SPIREA Bethlehamensis, 3-4 feet......... .75

Billardi, Billard's Spirea, 3-4 fcet.. ........ 65

callosa alba, Fortune's Spirea, 12-18 inches. ...

.60

$6.00 \quad 50.00$

$9.00 \quad 80.00$

callosa rubra, Fortune's Dwarf White Spirea

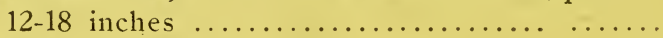

Froebelli, Frobel's Spirea

18-24 inches .......................... .65

multiflora arguta. $18-24$ inches .. .......

40.00

$5.50-45.00$

$5.00 \quad 40.00$

$4.50 \quad 36.00$

$\begin{array}{ll}4.50 & 36.00 \\ 5.00 & 40.00\end{array}$

opulifolia, Native Ninebark Spirea

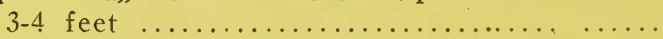

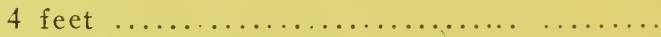

.60

$4.00 \quad 36.00$

.65

5.00

44.00

$5.00 \quad 40.00$

opulifolia aurea, Golden Spirea

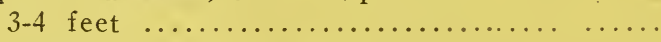

5 feet

.60

4.50

36.00

.65

5.00

40.07

prunifolia, Plum-leaved Spirea

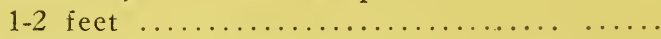

40.00

sorbifolia, 4sh leaved Spirea, 2-3 feet.......

Thunbergii, Snow Garland

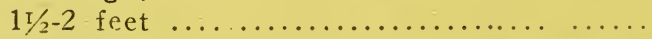

tomentosa, Native Steeple-bush or Hardhack

$2-3$ feet ............................ .70

Van Houttei, Van Houtte's Spirea (Bridal Wreath)

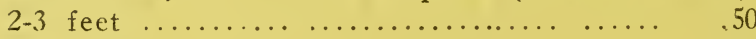

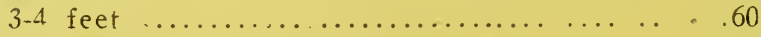

$4-5$ feet $\ldots \ldots \ldots \ldots \ldots \ldots \ldots \ldots \ldots \ldots \ldots \ldots . . . .6 . .70$

SYMPHORICARPUS racemosus, Native Snowberry

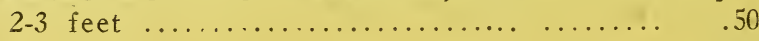

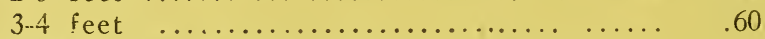

vulgaris, Native Indian Currant, Coral Berry

$2-3$ feet $\ldots \ldots \ldots \ldots \ldots \ldots \ldots \ldots \ldots \ldots \ldots \ldots \ldots \ldots$

vulgaris aurea, Golden leaved Indian Currant

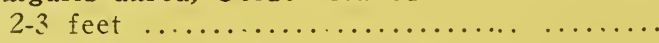

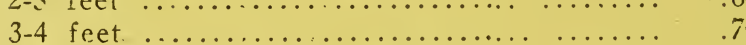

$\begin{array}{lll}60 & 4.50 \quad 40.00\end{array}$

$\begin{array}{lll}70 & 6.00 \quad 50.00\end{array}$

$60 \quad 5.00 \quad 40.00$

$6.00 \quad 45.00$

$3.50 \quad 30.00$

$5.00 \quad 36.00$

$6.00 \quad 50.00$

$\begin{array}{lll}50 & 4.00 \quad 30.00\end{array}$

$5.00 \quad 40.00$

$\begin{array}{lll}.50 & 3.50 \quad 30.00\end{array}$

$\begin{array}{lll}60 & 4.50 \quad 40.00\end{array}$

$\begin{array}{ll}70 & 5.50 \quad 45.00\end{array}$

SYRINGA Chinensis \& Japonica, Lilac, (see under Trees) Josikea, Hungarian I,lac

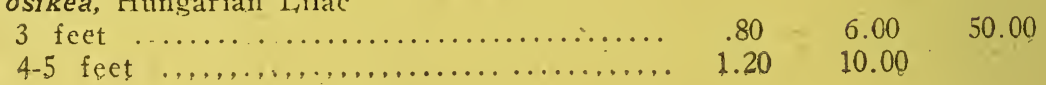


SYRINGA Continued

Persica, Persian Lilac

$3-4$ feet

persica alba, White Persian Lilac

3-4 feet

Rothomagensis, Rouen or Red Persian Lilac

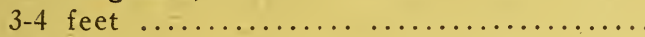

4-5 feet

villosa, Himalayan Lilac

2-3 feet

vulgaris, Common Lilac

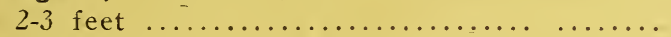

3-4 feet $\ldots \ldots \ldots \ldots \ldots \ldots \ldots \ldots \ldots \ldots \ldots \ldots \ldots \ldots$

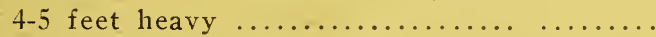

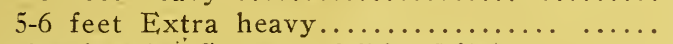

vulgaris alba, Common White Lilac

3-4 feet

SYRINGA in variety

Charles $X$, Single Deep Red, 3 feet

Emilie Lemoine, Double Rose Pink, 3 feet

Ludwig Spaeth, Single Dark Purple, 3 feet

Madame Lemoine, Double White, 2-3 feet

IMichael Buchner, Double Pale Lilac, 2-3 feet

Pres. Grevy, Double Rose Lilac, 2-3 feet

Princess Alexander, 2-3 feet

2-3 feet

3-4 feet

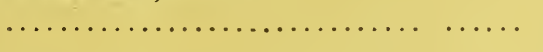

1.00

1.20

8.00

10.00

.80

hispida aestivalis, $3-4$ fect $\ldots \ldots \ldots \ldots \ldots \ldots . . . . . .$.
IBURNUM acerfolium, Native Dockmackie Arrow-wod

7.00

6.00

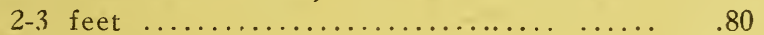

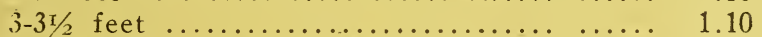

Americanum, American Highbush Cranberry

2-3 feet

6.50

9.00

6.50

3.00

6.00

$12-18$ incl

$2-2 \mathrm{r} / 2$ feet

cassinoides, Withe Rod

2-3 feet

dentatum, Native Arrow-wood

2-3 feet

lantana, American Wayfaring Tree,

3 feet

3.4. feet

lentago, Native Sweet Viburnum or Sheepberry

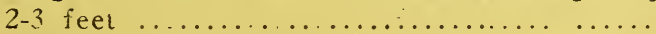

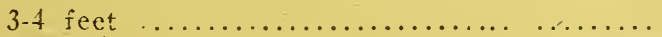

4-5 feet $\ldots \ldots \ldots \ldots \ldots \ldots \ldots \ldots \ldots \ldots \ldots \ldots \ldots$.

Molle, Soft leaved Viburnum, 2-3 feet ......

opulus, Common Highbush Cranberry

2-3 feet

5-6 feet

opulus sterilis, Common Snowball

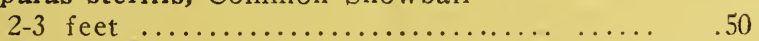

3-4 feet

25.00

50.00

7.00

60.00

$5.50 \quad 50.00$

5.00

40.00

$6.00 \quad 50.00$

$7.00^{\circ}$

60.00

$10.00 \quad 90.00$

$14.00 \quad 120.00$

.60

7.50

65.00

$4.00 \quad 35.00$

\begin{tabular}{l}
$1.00 \quad 9.00 \quad 80.00$ \\
\hline
\end{tabular}

prunifolium, Native Black Haw

3-4 feet 
VIBURNUM Cōntinued

tomentosum, 2-21/2 feet.

tomentosum var. plicatum, Japanese Snowball

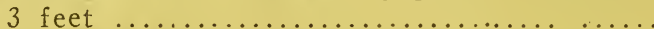

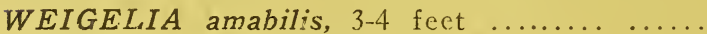

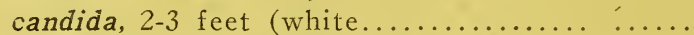

Desboisi, 3-4 feet. (Deep Innse) .............

Eva Rathke, 2.3 ieet. (Dark Red) ..........

3-4 feet

fioribunda, 3-4 feet (Dark Red)

rosea, 2-3 feet, Rose Colored Weigclia.......

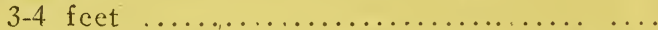

variegata, 3-4 feet, variegated leaved Weigelia

XANTHOXYLUM Americana, Native Northern

Prickley Ash

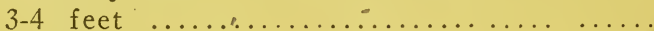

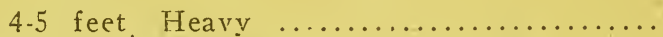

ZANTHORHIZA Apiifolio, $8-12$ inches........

\section{VINES}

ACTINIDA arguta, Japanese Silver Vine

2 year ............................ 75

$A K E B I A$ quinata, Five leaved Akebia

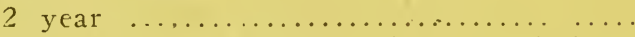

AMPELOPSIS Engelmanni, Engelmann's Ivy

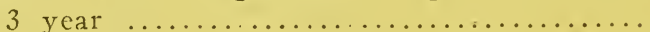

quinquefolia, Native Virginia Creeper

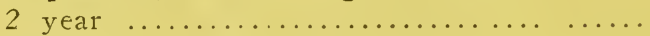

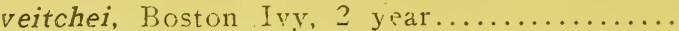

CELASTRUS articulatus, Japan Bittersweet

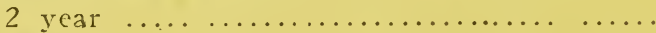

scadens, American Bittersweet, 2 year.......

CLEMATIS

Henryii, Large Creamy White, 2 year .......

Jackmanni, Violet Purple, 2 year............ paniculata, Japanese Virgin's Bower, 2 year. Virginiana, Common Native Virgin's Bower

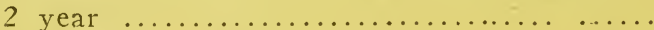

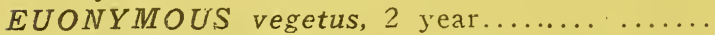

LONICERA flava, Yellow Trumpet Honeysuckle

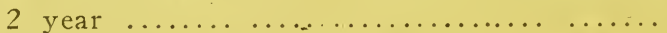

Halleana, Hall's Japan Honeysuckle

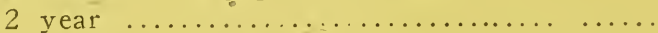

sempervirens, Scarlet Trumpet Honeysuckle

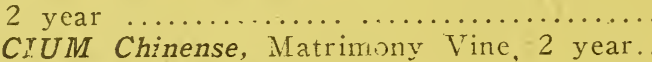

LYCrUM Chinense, Matrimony Vine, 2 year
PUERARIA Thunbergiani, Jack and the Bean

Stalk or Kudzu Vine

2 year $\ldots$ Kudzu Vine

TECOMA radicans, Trumpet Creeper

2 year ........................... 70

VINCA minor, Wintergreen, 2 year.......... $\quad .40$

VITIS aestivalis, Native Summer Grape

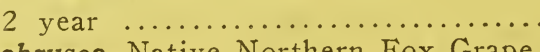

Labrusca, Native Northern Fox Grape

2 year
Each

.85

Per 10 Per 100

7.50

10.00

$1.25 \quad 11.00$

$.80 \quad 7.00$

$.80 \quad 7.00$

60.80

$.80 \quad 7.00$

60.00

.80

7.00

60.00

.90

7.50

60.00

.80

.65

.70

.70

7.00

5.00

6.00

70.00

60.00

40.00

6.00

50.00

50.00

.80

1.00

6.00

50.00

.60

8.00

4.50

40.00

Each

Per 10 Per 100

40.00

50.00 
WISTARIA frutescens, American Wistaria Each Per 10 Per 100

3 year ................................. $80 \quad 6.00 \quad 55.00$

sinensis, Chinese Biue Wistaria, 2 year..... $\quad 80 \quad 6.00 \quad 50.00$

sinensis alba, Chinese White Wistaria

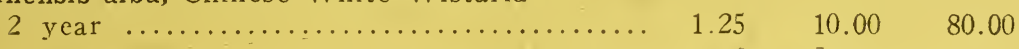

\section{PERENNIALS}

FIELD GROWN. ONE YEAR, EXCEPT WHERE NOTED.

Achillea ............The Pearl, 2 yr........... $\$ .20 \$ 1.80 \$ 16.00$ Milfoil

Aconitum ............Napellus ..................50 4.00

Monkshood

Alyssum ............ Rostratum ...............25 2.00

Madwort

Anchusa ........... Ttalica, Dropmore ......... .25 2.20.20.00

Alkanet

Anthemis .............elwayii ...............20 $1.80 \quad 14.00$

Marguerite

Aquilegia ............Chrysantha, 2 yr. ........... $25 \quad 2.00 \quad 18.00$

Columbine Chrysantha Grandiflora Alba $.25 \quad 2.00 \quad 18.00$

Coerulea, 2' yr .............25 $2.00 \quad 16.00$

Mrs. Scott Elliott's strain,

2. yr $\ldots \ldots \ldots \ldots \ldots \ldots \ldots .25 \quad 2.40 \quad 20.00$

Rose Queen, 2 yr $\ldots \ldots \ldots \ldots .25 \quad 2.00 \quad 20.00$

Vulgaris fl. pl., 2 yr..........25 $2.00 \quad 16.00$

Areneria .............Montana ................ $25 \quad 2.00$

Sandwort

Aster ............... Alpina, blue, white ........22 $2.00 \quad 18.00$

Asters .............Climax, lavender, blue..... .30 $2.80 \quad 26.00$

Michaelmas Daisy Novae Angliae, rubra ...... $.25 \quad 2.50 \quad 24.00$

Novae Angliae, rosea rose.. $.25 \quad 2.50 \quad 24.00$

Robert Parker, loft lavender $.25 \quad 2.50 \quad 24.00$

St. Edgwin, beautiful pink

flowers ..................25 $25.50 \quad 24.00$

White Queen, large white... $.25 \quad 2.50 \quad 24.00$

Aubretia ............. Craeca, violet blue .........35 3.00

Baptisia ............Australis, 2 yr $\ldots \ldots \ldots \ldots . .25 \quad 2.40 \quad 20.00$

False Indigo

Bellis................Monstrous Pink ...........20 $1.50 \quad 12.00$

English Daisies White ...................20 $1.50 \quad 12.00$

Bocconia ............Cordata, 2 yr .............25 $2.00 \quad 16.00$

Plume Poppy

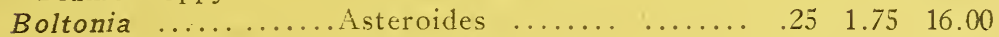

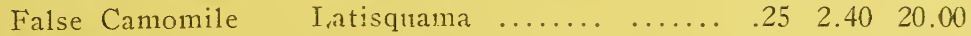

Campanula .........Carpatica, blue, 2 yr...... .25 $2.00 \quad 18.00$

Bell Flower $\quad$ Lactiflora $\ldots \ldots \ldots \ldots . \ldots \ldots . .25 \quad 2.00 \quad 18.00$

Medium blue, white, and rose $.20 \quad 2.00 \quad 18.00$

Medium Calycanthema,

white and rose $\ldots \ldots \ldots \ldots .25 \quad 2.00 \quad 18.00$

Persicifolia grandiflora al-

ba, 2 yr ..................25 $2.40 \quad 20.00$

Rotundifolia, 2 yr $\ldots \ldots \ldots . .25 \quad 2.40$

Rapunculoides, 2 yr $\ldots \ldots . .25 \quad 2.40$

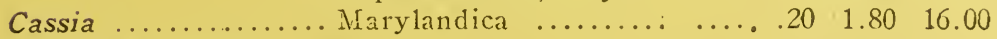

American Senna 
Catanache

Centaurea

.Coreulea, blue

$.201 .80 \quad 16.00$

Hardhea ............ Babylonica, tall yellow flow-

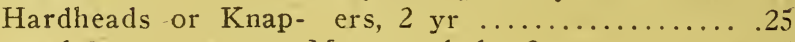

weed

Macroccphala, 2 yr .........25

Montana, blue .............25

Ruthenica, straw color ...... .25

$2.00 \quad 18.00$

2.00

2.00

Cerastium

Tomentosum ...............25

$2.00 \quad 18.00$

Snow in Summer

Cephalaria ...........Alpina

$25 \quad 2.00 \quad 18.00$

Chrysanthemum

Leucanthemum

Hardy Daisy

.Shasta Daisy, Alaska ...... $.201 .80 \quad 14.00$

Chrysanthemum

King Yidward VII ...........25 $25 \quad 2.00 \quad 16.00$

Cineraria

Hardy. large flowering, rose,

white, yellow, red........ $25 \quad 2.50 \quad 24.00$

Cineraria ...........Maritima, handsome whitened

foliage ................ $20 \quad 2.00$

Clemataquila

Kelway's Clemataquila or

Spurless Aquilagia ........ $.20 \quad 1.80$

Clemãtis

Integrifolia, $4 \mathrm{yr}$, strong.... $.25 \quad 2.40$

Integrifolia, 2 y r ............ $25 \quad 2.00$

Coreapsis

Lanceolata grand. .......... .20 1.80

14.00

Tickseed

Delphinium ............ Pelladona, 2 y r .......... 25

Perennial Larkspur Chinese Pumilum ........... 25

$2.40 \quad 20.00$

Formosum, 2 yr $\ldots \ldots \ldots \ldots \ldots .30$

Hybrids, English mixed,

$2 \mathrm{yr} \ldots \ldots \ldots \ldots \ldots \ldots \ldots \ldots \ldots \ldots \ldots \ldots \ldots .25$

$1.80 \quad 16.00$

$2.40 \quad 20.00$

Alba $\ldots \ldots \ldots \ldots \ldots \ldots \ldots \ldots, 20$

$2.40 \quad 20.00$

Dianthus Barbatus

Newport Pink ..............25

$1.80 \quad 16.00$

Sweet-William

Scarlet Beauty .............25

$2.00 \quad 16.00$

2.0016 .00

Dianthus Deltoides

. Rose ......................25

$2.00-16.00$

Maiden Pink

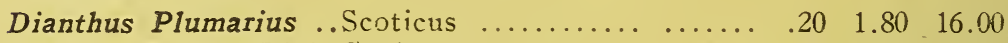

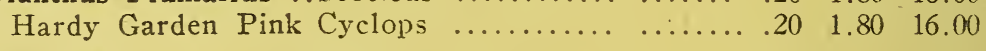

Dicentra ........... Eximia $\ldots \ldots \ldots \ldots \ldots . . . \ldots . .40$

Bleeding Heart

Dictamnus .........Alba, Pure white .......... $35 \quad 3.20 \quad 30.00$

Gas Plant

Franxinell, Showy rosy pink

with deeper veins ....... .35

$3.20 \quad 30.0$

Digitalis Gloxiniaeflora, white and

Foxglove

rose $\ldots \ldots \ldots \ldots . . . \ldots \ldots .20$

Grandiflora, 2 yr $\ldots \ldots \ldots \ldots . .20$

Lanata. $2 \mathrm{yr} \ldots \ldots \ldots \ldots \ldots \ldots . .20$

$\begin{array}{ll}1.80 & 16.00\end{array}$

$1.80 \quad 16.00$

Ritro, 2 yr .............25

1.8016 .00

Echinops

$2.40 \quad 20.00$

Globe Thistle

Eryngium ..........Amethystinum, 2 y r $\ldots \ldots \ldots .25 \quad 2.40 \quad 20.00$ Sea Holly

Eupatorium ........... Aseratoides, 2 yr $\ldots \ldots \ldots \ldots .20 \quad 1.80 \quad 14.00$

Thorough Wort

Gaillardia ............Kelway's Exhibition Strain.. .20 $1.80 \quad 14.00$ Blanket Flower

Galega ..............Hartlandi

$.25 \quad 2.00 \quad 16.00$

Goat's Rue 


\begin{tabular}{|c|c|c|c|}
\hline & Each & 10 & 100 \\
\hline \multirow[t]{2}{*}{ Grasses } & $\begin{array}{l}\text { Eulalia Japonica } \\
\text { Pliararis Arundinacea }\end{array}$ & 2.40 & \\
\hline & variegata ................. 20 & 2.00 & 15.00 \\
\hline Gypsophila & Acutifolia .. & 2.00 & 18.00 \\
\hline Baby's Breath & 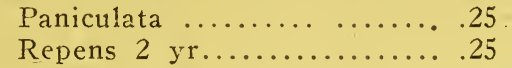 & $\begin{array}{l}2.20 \\
2.20\end{array}$ & $\begin{array}{l}20.00 \\
20.00\end{array}$ \\
\hline Sparsifolius ...... . ...... 20 & Sparsifolius $\ldots \ldots \ldots$. $\ldots \ldots \ldots$ & 1.80 & 14.00 \\
\hline $\begin{array}{l}\text { Heliopsis } \\
\text { Orange Sunflower }\end{array}$ & Pitcheriana 2 yr.............. & 1.80 & 16.00 \\
\hline Hemerocallis ...... & Flava & 2.20 & 20.00 \\
\hline Yellow Day Lily & $\begin{array}{l}\text { Fulva heavy } \ldots \ldots \ldots \ldots \ldots \\
\text { Thunbergii } \ldots \ldots \ldots\end{array}$ & $\begin{array}{l}2.00 \\
2.20\end{array}$ & $\begin{array}{l}18.00 \\
20.00\end{array}$ \\
\hline \multicolumn{2}{|c|}{$\begin{array}{l}\text { Hesperis } \ldots . . . . . . . . M a t r o n a l i s ~ \\
\text { Sweet Rocket }\end{array}$} & 2.00 & 14.00 \\
\hline \multirow{5}{*}{$\begin{array}{c}\text { Hibiscus } \\
\text { Mallow } \\
\text { Hollyhock }\end{array}$} & . Mallow Marvels mixed...... .25 & 2.40 & 20.00 \\
\hline & $\begin{array}{l}\text { Double, white, pink, crimson } \\
\text { yellow, maroon, salmon } .20\end{array}$ & 1.80 & 14.00 \\
\hline & $\begin{array}{l}\text { Newport Pink ............... } 20 \\
\text { Allegheny fringed mixed }\end{array}$ & 1.50 & 14.00 \\
\hline & $\begin{array}{l}\text { colors } \ldots \ldots \ldots \ldots \ldots \ldots \\
\text { Single mixed } 2 \\
\text { yr................ }\end{array}$ & $\begin{array}{l}2.00 \\
2.00\end{array}$ & $\begin{array}{l}14.00 \\
16.00\end{array}$ \\
\hline & Sempervirens $\ldots \ldots \ldots \ldots \ldots .20$ & 1.80 & 16.00 \\
\hline $\begin{array}{l}\text { Iberis } \ldots . . . . . . . . . \\
\quad \text { Hardy Candytuft }\end{array}$ & Gibraltarica ........ & 2.00 & \\
\hline \multicolumn{4}{|c|}{ 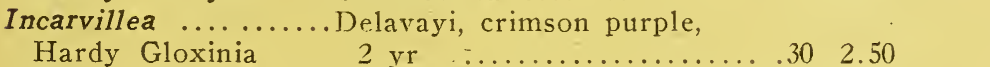 } \\
\hline \multirow{3}{*}{ Inula $\ldots \ldots \ldots$} & Ensifolia, 2 yr $\ldots \ldots \ldots \ldots \ldots \ldots . .25$ & 2.00 & \\
\hline & Glandulosa superba, 2 yr.... 25 & 2.00 & \\
\hline & Strong divisions & & \\
\hline \multirow{9}{*}{ Iris Germanica } & Aurea, Rich Chrome yellow...20 & 2.00 & 18.00 \\
\hline & Celeste, pale azure blue .... .20 & 1.80 & 15.00 \\
\hline & Flavescens, soft yellow .... .20 & 1.80 & $14 . \mathrm{CO}$ \\
\hline & $\begin{array}{l}\text { Florentina alba, white ...... } 20 \\
\text { Hèr Majesty, rose pink and }\end{array}$ & 1.80 & 14.00 \\
\hline & $\begin{array}{l}\text { crimson ..................... } 25 \\
\text { Honorabilis, golden yellow }\end{array}$ & 2.00 & 18.00 \\
\hline & $\begin{array}{l}\text { and Mahogany brown ...... } 20 \\
\text { Mad. Chereau, white edged }\end{array}$ & 1.80 & 14.00 \\
\hline & $\begin{array}{l}\text { withb lue ................. } .20 \\
\text { Pallida Dalmatica, extra }\end{array}$ & 1.80 & 14.09 \\
\hline & fine lavender $\ldots . . . \cdots \cdots \cdots, .35$ & 3.00 & \\
\hline & 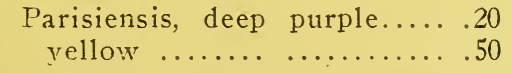 & 1.80 & 14.00 \\
\hline \multirow{5}{*}{$\begin{array}{c}\text { Iris } \\
\text { Iris } \\
\text { Kaempferi } \\
\\
\end{array}$} & $\begin{array}{l}\text { Japan Iris, mixed, } 2 \text { yr...... } \\
\text {.Cristata, Crested Iris, light }\end{array}$ & 2.40 & \\
\hline & blite $\ldots \ldots \ldots \ldots \ldots \ldots .25$ & 2.00 & 16.00 \\
\hline & Ochrolcuca, 2 yr & 2.40 & \\
\hline & $\begin{array}{l}\text { Pumila hybrids, dwarf....... } .20 \\
\text { Pseudacorus, yellow water }\end{array}$ & 1.80 & 14.00 \\
\hline & flag ....................... & 2.00 & 14.00 \\
\hline \multirow[t]{5}{*}{ Iris } & . Siberian blue ..............25 & 1.80 & 14.00 \\
\hline & Siberian Snow Queen ...... 25 & 2.00 & 16.00 \\
\hline & Siberian mixed ............. 25 & 2.00 & 14.00 \\
\hline & Sanguinea $\ldots \ldots \ldots \ldots \ldots \ldots .25$ & 2.00 & 14.00 \\
\hline & Verna, dwarf Iris, violet, blue, 25 & 2.00 & 16.00 \\
\hline
\end{tabular}




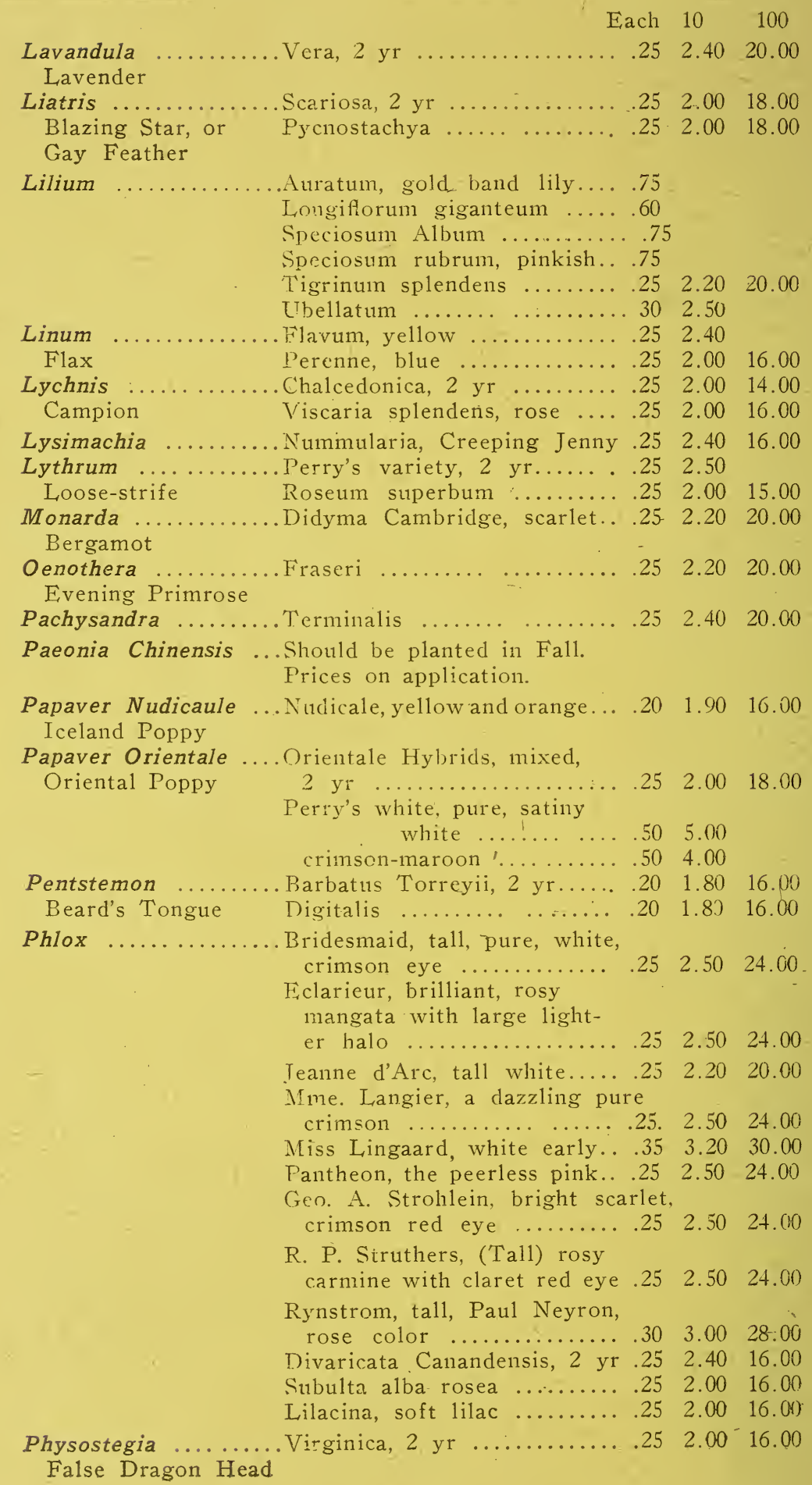




\begin{tabular}{|c|c|c|c|}
\hline & Each & 10 & 100 \\
\hline Platycodon & Mariesi, 2 yr, blue... & 1.80 & 16.00 \\
\hline Balloon Flower & Mariesi alba, white ..... & 1.80 & 16.00 \\
\hline $\begin{array}{l}\text { Polemonium } \ldots . . . \\
\quad \text { Greek Valerian }\end{array}$ & . Richardsoni & 2.00 & 18.00 \\
\hline Primula veris .. & Giant Polyanthus & 2.00 & 18.00 \\
\hline Pyrethrum .. & . Hydridum Kelways, 2 yr.... .25 & 2.20 & 20.00 \\
\hline & Hydridum Kelways, 1 yr... .20 & 1.80 & 16.00 \\
\hline Rudbeckia & . Golden Glow ..............25 & 2.00 & 18.00 \\
\hline Cone Flower & $\begin{array}{l}\text { Newmanni } \ldots \ldots \ldots \text {. } \\
\text { Purpurea, } 2 \text { yr, purple } \\
\text { cone flower } \ldots \ldots \ldots \ldots \ldots \ldots\end{array}$ & 2.00 & 16.00 \\
\hline Salvia & . Azurea grandiffora, 1 yr...... 20 & 1.80 & 15.00 \\
\hline Meadow Sage & Azurea grandiflora, 2 yr.... .25 & 2.00 & 16.00 \\
\hline Saponaria ...... &. Ocymoides $\ldots \ldots \ldots$.........25 & 2.00 & 16.00 \\
\hline Scabiosa & .Caucasica .................25 & & \\
\hline Blue Bonnet & $\ldots \ldots \ldots \ldots \ldots .25$ & 2.00 & \\
\hline Sedum $\ldots . . .$. & Acre $\ldots \ldots \ldots \ldots \ldots \ldots \ldots \ldots .20$ & 1.80 & \\
\hline Stone-crop & Spectabile & 2.00 & 18.00 \\
\hline $\begin{array}{l}\text { Silene } \ldots \ldots \ldots \\
\quad \text { Catchfly }\end{array}$ & . Schafta $\ldots$ & 2.00 & 18.00 \\
\hline Solidago ....... & Golden , Rod ...... & 2.00 & 18.00 \\
\hline Spirea & Filipendula, 2 yr. & 2.00 & 18.00 \\
\hline $\begin{array}{l}\text { Goat's Beard, } \\
\text { Meadow Sweet }\end{array}$ & (Tlmaria, 2 yr $\ldots \ldots \ldots \ldots \ldots \ldots .25$ & 2.00 & 18.00 \\
\hline $\begin{array}{l}\text { Stachys } \ldots . . . . . \\
\text { Woundwort }\end{array}$ & . Lanata, 2 yr $\ldots \ldots \ldots \ldots \ldots . .20$ & 1.80 & 16.00 \\
\hline Statice & Iatifolia & 2.40 & 20.00 \\
\hline Sea Lavender & Tartarica & 2.40 & 20.00 \\
\hline Stokesia ...... & Cyanea blue ...... & 2.20 & 20.00 \\
\hline Stokes' Aster & Cyanea alba .............. 25 & 2.20 & 20.00 \\
\hline Thalictrum & Acliantifolia, 2 yr ..........25 & 2.00 & 18.00 \\
\hline Meadow Rue & 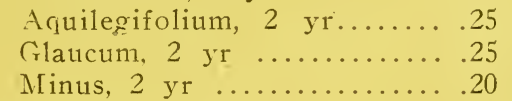 & $\begin{array}{l}2.50 \\
2.50 \\
2.00\end{array}$ & 20.00 \\
\hline Thermopsis & .Carolinaiana $\ldots \ldots \ldots \ldots \ldots . .25$ & 2.00 & 16.00 \\
\hline $\begin{array}{l}\text { Tradescantia ... } \\
\text { Spider Wort }\end{array}$ & …........ 20 & 1.80 & \\
\hline $\begin{array}{l}\text { Valeriana } \\
\text { Valerian }\end{array}$ & 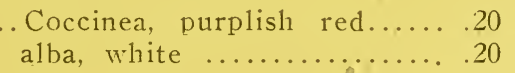 & $\begin{array}{l}1.80 \\
1.80\end{array}$ & $\begin{array}{l}16.00 \\
16.00\end{array}$ \\
\hline Veronica & Amethystima & 2.00 & 16.00 \\
\hline Speedwell & $\begin{array}{l}\text { Jongifolia Subsessilis } \ldots \ldots \ldots \\
\text { Spicta, } 2 \text { yr } \ldots \ldots \ldots \ldots \ldots \ldots \ldots \\
\end{array}$ & $\begin{array}{l}2.50 \\
2.20\end{array}$ & $\begin{array}{l}24.00 \\
20.00\end{array}$ \\
\hline $\begin{array}{l}\text { Vinca } \ldots . . . \ldots . . . \\
\text { Green Myrtle }\end{array}$ & Minor $\ldots \ldots \ldots \ldots . . . \ldots \ldots \ldots . .25$ & 2.00 & 18.00 \\
\hline $\begin{array}{l}\text { Viola Odorata .. } \\
\text { Violet }\end{array}$ & Semperflorens & 2.20 & 20.00 \\
\hline $\begin{array}{l}\text { Viola Cornuta } \\
\text { Tufted Pansies }\end{array}$ & Blue and yellow & 2.00 & 16.00 \\
\hline Yucca & Filamentosa, 3 yr. & 2.50 & 24.00 \\
\hline Adam's Needle & $\begin{array}{l}\text { Filamentosa, } 2 \text { yr. ........... } .25 \\
\text { Glanc'a, American native }\end{array}$ & 2.20 & 20.00 \\
\hline
\end{tabular}






\section{NAPERVILLE NURSERIES}

\section{NAPERVILLE, ILLINOIS}

GROUNDS AND OFFICE One-half mile west of the City of Naperville, Ill., on the Chicago-Aurora and Naperville-Oswego-Joliet Roads.

TELEPHONE-Bell System-Long Distance, Naperviile, No. 1.

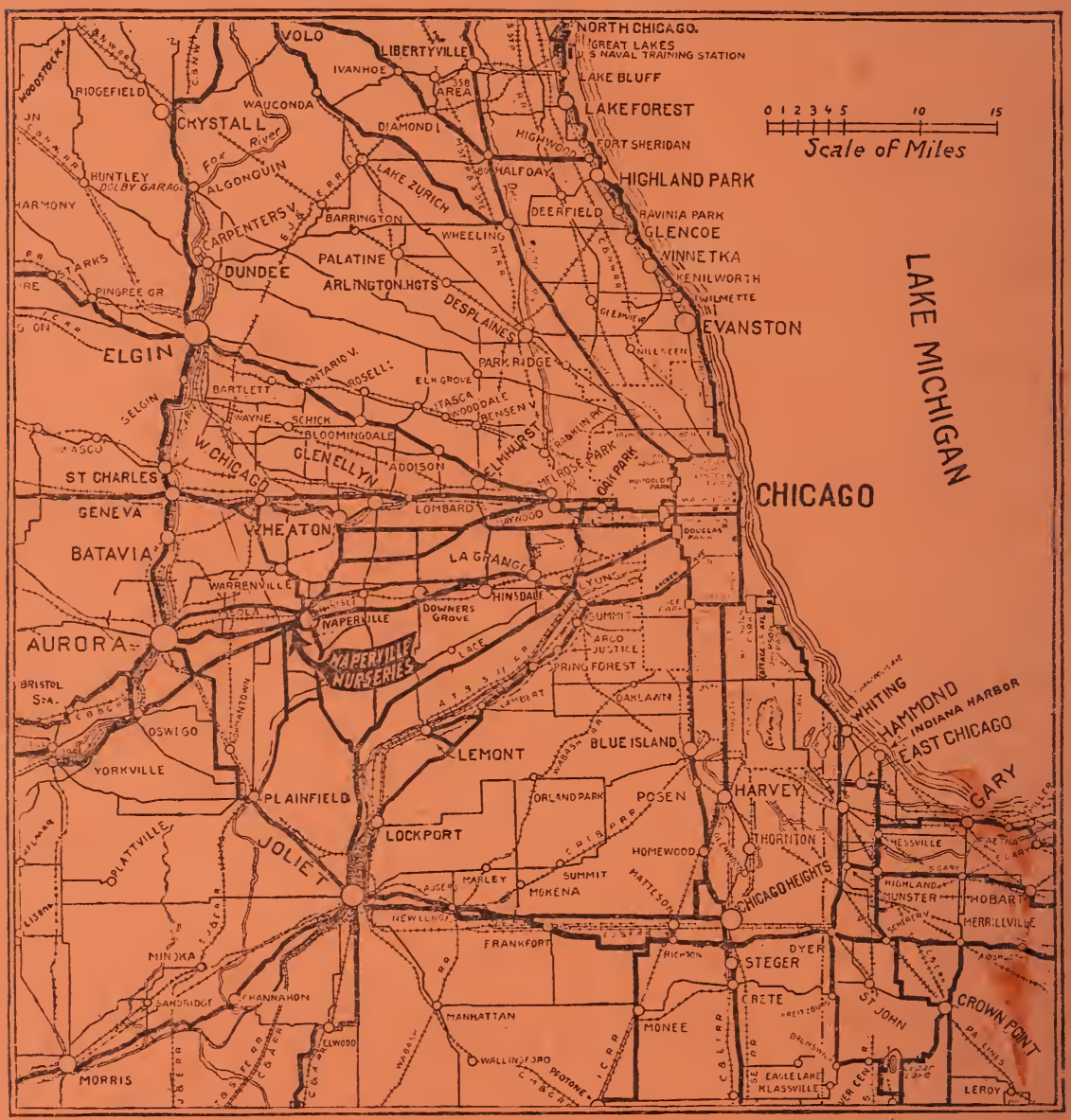

OUR NURSERIES are situated adjacent to the City of Naperville, DuPage County. Illinois, some 28 miles 11 est of Chicago, on the Chicago, Burlington \& Quincy Railroad. Popular automobile routes as the "Cannon Ball" and "Starved Rock" Trails pass our nurseries over designated State Roads.

FRUIT, SHADE AND ORNAMENTAL TREES AND SHRUBS PERENNIALS, EVERGREENS, ETC. 